\title{
Theoretical analysis of mixing in liquid clouds - Part 3: Inhomogeneous mixing
}

\author{
Mark Pinsky $^{1}$, Alexander Khain ${ }^{1}$, and Alexei Korolev ${ }^{2}$ \\ ${ }^{1}$ Department of Atmospheric Sciences, The Hebrew University of Jerusalem, Jerusalem, Israel \\ ${ }^{2}$ Environment Canada, Cloud Physics and Severe Weather Section, Toronto, Canada \\ Correspondence to: Alexander Khain (alexander.khain@mail.huji.ac.il)
}

Received: 26 August 2015 - Published in Atmos. Chem. Phys. Discuss.: 4 November 2015

Revised: 30 May 2016 - Accepted: 3 June 2016 - Published: 28 July 2016

\begin{abstract}
An idealized diffusion-evaporation model of timedependent mixing between a cloud volume and a dropletfree volume is analyzed. The initial droplet size distribution (DSD) in the cloud volume is assumed to be monodisperse. It is shown that evolution of the microphysical variables and the final equilibrium state are unambiguously determined by two non-dimensional parameters. The first one is the potential evaporation parameter $R$, proportional to the ratio of the saturation deficit to the liquid water content in the cloud volume, that determines whether the equilibrium state is reached at $100 \%$ relative humidity, or is characterized by a complete evaporation of cloud droplets. The second parameter $D a$ is the Damkölher number equal to the ratio of the characteristic mixing time to the phase relaxation time. Parameters $R$ and $D a$ determine the type of mixing.

The results are analyzed within a wide range of values of $R$ and $D a$. It is shown that there is no pure homogeneous mixing, since the first mixing stage is always inhomogeneous. The mixing type can change during the mixing process. Any mixing type leads to formation of a tail of small droplets in DSD and, therefore, to DSD broadening that depends on $D a$. At large $D a$, the final DSD dispersion can be as large as 0.2. The total duration of mixing varies from several to 100 phase relaxation time periods, depending on $R$ and $D a$.

The definitions of homogeneous and inhomogeneous types of mixing are reconsidered and clarified, enabling a more precise delimitation between them. The paper also compares the results obtained with those based on the classic mixing concepts.
\end{abstract}

\section{Introduction}

Cloud physics typically investigates two types of turbulent mixing: homogeneous and extremely inhomogeneous (e.g., Burnet and Brenguier, 2007; Andrejczuk et al., 2009; Devenish et al., 2012; Kumar et al., 2012). The concept of extremely inhomogeneous mixing in clouds was introduced by Latham and Reed (1977), Baker and Latham (1979), Baker et al. (1980) and Blyth et al. (1980). According to this concept, mixing of cloud air and sub-saturated air from cloud surrounding results in complete evaporation of a fraction of cloud droplets, whereas the size of other droplets remain unchanged. The studies of extremely inhomogeneous mixing were closely related to investigation of different mechanisms underlying enhanced growth of cloud droplets and warm precipitation formation (Baker et al., 1980; Baker and Latham, 1982). The concept of homogeneous mixing suggests that all the droplets partially evaporate, so the liquid water content decreases while the droplet concentration remains unchanged (Lehmann et al., 2009; Pt1). The significance of the concepts of homogeneous and inhomogeneous mixing goes far beyond formation of large-sized droplets. In fact, these concepts are closely related to the mechanisms involved in formation of droplet size distributions (DSD) in clouds and to the description of this formation in numerical cloud models. A detailed analysis of the classical concepts of homogeneous and extremely inhomogeneous mixing is given by Korolev et al. (2016, hereafter Pt1).

Mixing in clouds includes two processes: mechanical mixing caused by turbulent diffusion and droplet evaporation accompanied by increasing relative humidity. The relative contribution of these processes can be evaluated by compari- 
son of two characteristic timescales: the characteristic mixing timescale $\tau_{\text {mix }} \sim L^{2 / 3} \varepsilon^{-1 / 3}$ (where $L$ is the characteristic linear scale of an entrained volume and $\varepsilon$ is the dissipation rate of turbulent kinetic energy) and the time of phase relaxation $\tau_{\mathrm{pr}}=(4 \pi D \bar{r} N)^{-1}$ (where $N$ is droplet concentration in a cloud volume, $\bar{r}$ is the mean droplet radius and $D$ is the diffusivity of water vapor) characterizing the response of the droplet population to changes in humidity (the list of notations is given in Appendix). The choice of the phase relaxation time as the characteristic timescale of mixing is discussed by Pinsky et al. (2016) (hereafter referred to as Pt2) and will be further elaborated below.

Mixing is considered homogeneous if $\tau_{\text {mix }} / \tau_{\text {pr }} \ll 1$. At the first stage of mixing, the initial gradients of the microphysical and thermodynamic variables rapidly decrease to zero. By the end of this stage, the fields of temperature, humidity (hence, the relative humidity, $\mathrm{RH}$ ) and droplet concentration are spatially homogenized and all the droplets within the mixing volume experience the same saturation deficit. During the relatively lengthy second stage, droplets evaporate and increase the relative humidity in the volume. It was shown that homogeneous mixing takes place at scales below about $0.5 \mathrm{~m}(\mathrm{Pt} 2)$.

At spatial scales larger than $\sim 0.5 \mathrm{~m}, \tau_{\text {mix }} / \tau_{\mathrm{pr}}>1$ and the spatial gradients of RH remain for a long time. Consequently, droplets within the mixing volume experience different subsaturations, thus the mixing is considered inhomogeneous. At $\tau_{\text {mix }} / \tau_{\text {pr }} \gg 1$, the mixing is considered extremely inhomogeneous.

According to the classical conceptual scheme, during the first stage of extremely inhomogeneous mixing a fraction of droplets is transported into the droplet-free entrained volume and evaporates completely. The evaporation continues until the evaporating droplets saturate the initially dropletfree volume. At the second stage, turbulent mixing between the cloud volume and the initially droplet-free (but already saturated) volume homogenizes the gradients of droplet concentration and other quantities. Since both volumes are saturated, mixing does not affect droplet sizes. As a result, the final (equilibrium) state is characterized by the relative humidity $\mathrm{RH}=100 \%$ and the DSD shape similar to that before mixing, but with a lower droplet concentration. The same result (a decrease in droplet concentration but unchanged droplet size) is expected in cases of both monodisperse and polydisperse initial DSD. Since the DSD shape does not change, the characteristic droplet sizes (i.e., the mean square radius, the mean volume radius and the effective radius) do not change either in the course of extremely inhomogeneous mixing.

Thus, according to the classical concepts, the final equilibrium state with $\mathrm{RH}=100 \%$ is reached either by a partial evaporation of all droplets (homogeneous mixing) or a total evaporation of a certain portion of droplets that does not affect the remaining droplets (extremely inhomogeneous mixing) (Lehmann et al., 2009; Pt1).
In analyses of in situ measurements, the observed data are usually compared with those expected at the final state of mixing as assumed by the classical mixing concepts. If droplet concentration decreases without a corresponding change in the characteristic droplet radius, the mixing is considered "extremely inhomogeneous". If the characteristic droplet radius decreases with an increase of the dilution level while droplet concentration decreases insignificantly, the mixing is identified as "homogeneous". If both the characteristic droplet radius and the droplet concentration change, the mixing is considered as "intermediate". Quantitative evaluations of the microphysical processes specific for intermediate mixing remain largely uncertain.

As was discussed in $\mathrm{Pt} 2$, the final states of mixing suggested by the classical concepts are only hypothetical. To understand the essence of the final equilibrium states of mixing and evaluate the time needed to reach them, it is necessary to consider the time evolution of DSD in the course of mixing process. Time-dependent process of homogeneous mixing was analyzed in $\mathrm{Pt} 2$. It was shown that in important cases of wide polydisperse initial DSDs, the final state substantially differs from that hypothesized by the classical concepts.

In this study, which is a Pt3 of the set of studies, we analyze the time-dependent process of inhomogeneous mixing. The structure of the paper is as follows. The main concept and the basic equations for time-dependent inhomogeneous mixing are described in Sect. 2. Analysis of non-dimensional diffusion-evaporation equations is presented in Sect. 3. The design and the results of simulations of non-homogeneous mixing are outlined in Sects. 4 and 5. A discussion clarifying the concepts of homogeneous and inhomogeneous mixing is presented in concluding Sect. 6.

\section{The main concept and the basic equations}

During mixing of cloud volume and entrained air volume, the following two processes determine the change of the microphysical and thermodynamical variables: turbulent diffusion resulting in mechanical smoothing of the gradients of temperature, water vapor and droplet concentration, and droplet evaporation accompanied by phase transformation. In this study, inhomogeneous mixing is investigated based on the analysis and solution of a 1-D diffusion-evaporation equation. To our knowledge, the idea of using a diffusive model of turbulent mixing to describe the mixing process was first proposed by Baker and Latham (1982). A diffusion-evaporation equation was also analyzed by Jeffery and Reisner (2006). In order to get a more precise understanding of the physics of the mixing process the analysis is performed under the following main simplifying assumptions:

a. Turbulent mixing is analyzed neglecting vertical motions of mixing volumes, droplet collisions and droplet sedimentation. 


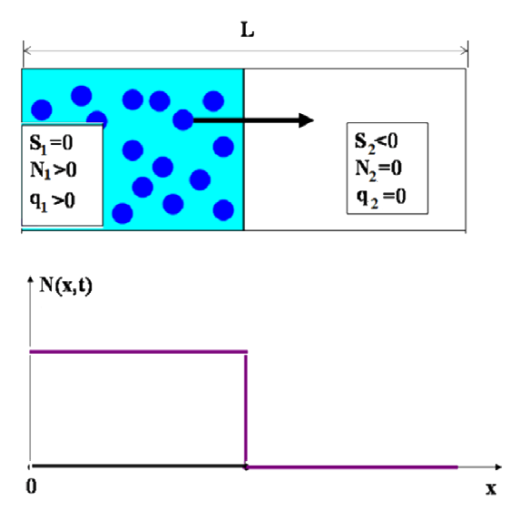

Figure 1. The schematic illustration of the 1-D mixing problem considered in the study. The initial state at $t=0$ is illustrated. The left volume of length $L / 2$ is a saturated cloudy volume; the right volume is a non-saturated air volume from the cloud environment.

b. The total mixing volume is assumed adiabatic.

c. Mixing is assumed to take place only along the $x$ direction, i.e., a 1-D task is considered;

d. The initial DSD in the cloud volume is assumed monodisperse.

Other assumptions and simplifications are discussed below.

A schematic illustration of the initial conditions used in the study is shown in Fig. 1. Two air volumes are assumed to mix: a cloud volume (left) and a droplet-free volume (right), each having the linear size of $L / 2$. The value of $L$ is assumed within the range of several tens to a few hundred meters. The mixing starts at $t=0$. The cloud volume is initially saturated $S_{1}=0$, the initial droplet concentration is $N_{1}$ and the initial liquid water mixing ratio is $q_{1}=\frac{4 \pi \rho_{\mathrm{w}}}{3 \rho_{\mathrm{a}}} N_{1} r_{0}^{3}$. In the droplet-free volume the initial conditions are $\mathrm{RH}_{2}<100 \%$ (i.e., $S_{2}<0$ ), $N_{2}=0$ and $q_{2}=0$. Therefore, the initial profiles of these quantities along the $x$ axis are step functions

$N(x, 0)= \begin{cases}N_{1} & \text { if } 0 \leq x<L / 2 \\ 0 & \text { if } L / 2 \leq x<L\end{cases}$

$S(x, 0)= \begin{cases}0 & \text { if } 0 \leq x<L / 2 \\ S_{2} & \text { if } L / 2 \leq x<L\end{cases}$

$q(x, 0)= \begin{cases}q_{1} & \text { if } 0 \leq x<L / 2 \\ 0 & \text { if } L / 2 \leq x<L\end{cases}$

The initial profile of droplet concentration is shown in Fig. 1. In this study, averaged equations are used. We do not consider mixing at scales below several millimeters. At the scales of averaging, there exist clear definitions of droplet concentration, supersaturation and other "macro scale" quantities. The mixing is assumed to be driven by isotropic turbulence within the inertial sub-range where the Richardson's law is valid. Accordingly, turbulent diffusion (turbulent mixing) is described by a 1-D equation of turbulent diffusion with a turbulent coefficient $K$. The turbulent coefficient is evaluated as proposed by Monin and Yaglom (1975)

$K(L)=C \varepsilon^{1 / 3} L^{4 / 3}$.

In Eq. (2), $C$ is a constant. Equation (2) is valid in case turbulent diffusion is considered, i.e., at scales where molecular diffusion can be neglected.

Since the total mixing volume is adiabatic, the fluxes of different quantities through the left and right boundaries of the volume are equal to zero at any time instance, i.e.,

$$
\begin{aligned}
& \frac{\partial N(0, t)}{\partial x}=\frac{\partial N(L, t)}{\partial x}=0 ; \quad \frac{\partial q(0, t)}{\partial x}=\frac{\partial q(L, t)}{\partial x}=0 ; \\
& \frac{\partial q_{\mathrm{v}}(0, t)}{\partial x}=\frac{\partial q_{\mathrm{v}}(L, t)}{\partial x}=0,
\end{aligned}
$$

where $q_{\mathrm{v}}$ is the water vapor mixing ratio.

During mixing, droplets in the mixing volume experience different subsaturations, therefore, the initially monodisperse DSD will become polydisperse. The droplets that were transported into the initially droplet-free volume will undergo either partial or complete evaporation. The evaporation leads to a decrease in both droplet size and droplet concentration.

The basic system of equations that describes the processes of diffusion and of evaporation which occur simultaneously is to be derived. The first equation is written for value $\Gamma$ defined as

$\Gamma=S+A_{2} q$.

This value is conservative in a moist adiabatic process, i.e., it does not change during phase transitions (Pinsky et al., 2013, 2014). In Eq. (4), the coefficient $A_{2}=\frac{1}{q_{\mathrm{v}}}+\frac{L_{\mathrm{w}}^{2}}{c_{p} R_{\mathrm{v}} T^{2}}$ is a weak function of temperature that changes by $\sim 10 \%$ when temperatures change by $\sim 10^{\circ} \mathrm{C}$ (Pinsky et al., 2013). In this study, it is assumed that $A_{2}=$ constant. In Eq. (4), $q=\frac{4 \pi \rho_{\mathrm{w}}}{3 \rho_{\mathrm{a}}} \int_{0}^{\infty} r^{3} f(r) \mathrm{d} r$ is the liquid water mixing ratio and $f(r)$ is the DSD. The quantity $\Gamma$ obeys the diffusion equation

$$
\frac{\partial \Gamma(x, t)}{\partial t}=K \frac{\partial^{2} \Gamma(x, t)}{\partial x^{2}}
$$

with the boundary conditions $\frac{\partial \Gamma(0, t)}{\partial x}=\frac{\partial \Gamma(L, t)}{\partial x}=0$ and the initial profile at $t=0$

$$
\Gamma(x, 0)= \begin{cases}A_{2} q_{1} & \text { if } 0 \leq x<L / 2 \\ S_{2} & \text { if } L / 2 \leq x<L\end{cases}
$$

Therefore, function $\Gamma(x, 0)$ is positive in the left volume, and negative in the right volume.

Since $\Gamma$ does not depend on phase transitions, Eq. (5) can be solved independently of other equations. The solution of 


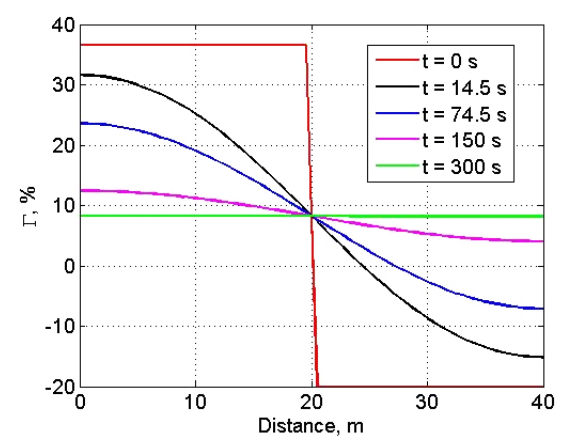

Figure 2. An example of $\Gamma(x, t)$ evolution during mixing.

Eq. (5) with initial conditions (Eq. 6) is (Polyanin and Zaitsev, 2004)

$$
\begin{aligned}
\Gamma(x, t) & =\sum_{n=0}^{\infty} a_{n} \exp \left(-\frac{K n^{2} \pi^{2} t}{L^{2}}\right) \cos \left(\frac{n \pi x}{L}\right) \\
& =\frac{1}{2}\left(S_{2}+A_{2} q_{1}\right)+\left(A_{2} q_{1}-S_{2}\right) \\
& \sum_{n=1}^{\infty} \frac{\sin (n \pi / 2)}{n \pi / 2} \exp \left(-\frac{K n^{2} \pi^{2} t}{L^{2}}\right) \cos \left(\frac{n \pi x}{L}\right),
\end{aligned}
$$

where the Fourier coefficients of expanding the step function (Eq. 6) are

$$
\begin{aligned}
& a_{0}=\frac{1}{2}\left(A_{2} q_{1}+S_{2}\right) \\
& a_{n}=\left(A_{2} q_{1}-S_{2}\right) \frac{\sin (n \pi / 2)}{n \pi / 2}, n=1,2, \ldots
\end{aligned}
$$

An example of spatial dependencies of $\Gamma(x, t)$ at different time instances during the mixing is shown in Fig. 2. One can see a decrease in the initial gradients and a tendency to establish a horizontally uniform value of $\Gamma$. Since the initial volume was divided into two equal parts, the diffusion leads to formation of a constant limit value of function $\Gamma(x, \infty)=\frac{1}{2}(\Gamma(0,0)+\Gamma(L, 0))$.

The second basic equation is the equation for diffusional droplet growth, taken in the following form (Pruppacher and Klett, 2007):

$\frac{\mathrm{d} \sigma}{\mathrm{d} t}=\frac{2 S}{F}$

where $\sigma=r^{2}$ is the square of droplet radius and $F=$ $\frac{\rho_{\mathrm{w}} L_{\mathrm{w}}^{2}}{k_{\mathrm{a}} R_{\mathrm{V}} T^{2}}+\frac{\rho_{\mathrm{W}} R_{\mathrm{v}} T}{e_{s}(T) D}$. The value of coefficient $F$ is considered constant in this study. The solution of Eq. (9) is

$\sigma(t)=\frac{2}{F} \int_{0}^{t} S\left(t^{\prime}\right) \mathrm{d} t^{\prime}+\sigma_{0}$.

The third main equation describes the evolution of DSD. In the following discussion, the DSD will be presented in the form $g(\sigma)$ which is the distribution of the square of the radius. This formulation directly utilizes the property of the diffusion growth equation (Eq. 9) according to which the time changes of DSD are reduced to shifting the distributions in the space of square radii, while the shape of the distribution remains unchanged. The standard DSD $f(r)$ is related to $g(\sigma)$ as $f(r)=2 r \cdot g\left(r^{2}\right)$.

The normalized condition for $g(\sigma)$ is

$N=\int_{0}^{\infty} g(\sigma) \mathrm{d} \sigma$

where $N$ is the droplet concentration. Using DSD $g(\sigma)$, the liquid water mixing ratio can be presented as integral

$q=\frac{4 \pi \rho_{\mathrm{w}}}{3 \rho_{\mathrm{a}}} \int_{0}^{\infty} \sigma^{3 / 2} g(\sigma) \mathrm{d} \sigma$.

The 1-D diffusion-evaporation equation for the nonconservative function $g(\sigma)$ can be written in the form (Rogers and Yau, 1989)

$\frac{\partial g(\sigma)}{\partial t}=K \frac{\partial^{2} g(\sigma)}{\partial x^{2}}-\frac{\partial}{\partial \sigma}\left(\frac{\mathrm{d} \sigma}{\mathrm{d} t} g(\sigma)\right)$

where the first term on the right-hand side of Eq. (13) describes changes in the DSD due to spatial diffusion, while the second term on the right-hand side describes changes in the DSD due to evaporation. Substitution of Eq. (9) into Eq. (13) leads to the following equation

$\frac{\partial g(x, t, \sigma)}{\partial t}=K \frac{\partial^{2} g(x, t, \sigma)}{\partial x^{2}}-\frac{2 S(x, t)}{F} \frac{\partial g(x, t, \sigma)}{\partial \sigma}$.

To close Eq. (14), Eq. (4) should be used in the form

$S(x, t)=\Gamma(x, t)-A_{2} q(x, t)$,

where $q(x, t)$ is calculated according to Eq. (12). Equations $(12,14,15)$ constitute a closed set of equations allowing calculation of $g(x, t, \sigma)$.

To proceed to the equations for DSD moments, let us define a moment of DSD $g(\sigma)$ of order $\alpha$ as

$m_{\alpha}=\overline{\sigma^{\alpha}}=\int_{0}^{\infty} \sigma^{\alpha} g(\sigma) \mathrm{d} \sigma$.

Multiplying Eq. (14) by $\sigma^{\alpha}$, integrating within limits $[0 \ldots \infty]$ and assuming that $\sigma^{\alpha} g(\sigma) \rightarrow 0$ when $\sigma \rightarrow \infty$, yield a recurrent formula for the DSD moments

$\frac{\partial m_{\alpha}(x, t)}{\partial t}=K \frac{\partial^{2} m_{\alpha}(x, t)}{\partial x^{2}}+\alpha \frac{2 S}{F} m_{\alpha-1}(x, t)$.

Eq. (17) provides a recurrent relationship between the DSD moments of different orders. A similar relationship was discussed by Pinsky et al. (2014) while analyzing diffusion growth in an ascending adiabatic parcel. 
In particular, the equation for the liquid water mixing ratio that is a moment of the order of $\alpha=\frac{3}{2}$ can be written as

$$
\frac{\partial q(x, t)}{\partial t}=K \frac{\partial^{2} q(x, t)}{\partial x^{2}}+\frac{4 \pi \rho_{\mathrm{w}} N(x, t) \bar{r}(x, t)}{F \rho_{\mathrm{a}}} S(x, t),
$$

where the mean radius $\bar{r}(x, t)=\frac{m_{1 / 2}}{m_{0}}$.

In the general case, Eq. (18) is not closed, since concentration $N(x, t)$ and $\bar{r}(x, t)$ are unknown functions of time and spatial coordinates.

The characteristic time of evaporation and of supersaturation change is the phase relaxation time (Korolev and Mazin, 2003)

$\tau_{\mathrm{pr}}=\frac{\rho_{\mathrm{a}} F}{4 \pi \rho_{\mathrm{w}} A_{2} N \bar{r}}$.

Using Eq. (19), Eq. (18) can be rewritten as

$$
\begin{aligned}
\frac{\partial q(x, t)}{\partial t} & =K \frac{\partial^{2} q(x, t)}{\partial x^{2}}+\frac{1}{\tau_{\mathrm{pr}}(x, t)}\left[\frac{1}{A_{2}} \Gamma(x, t)-q(x, t)\right] \\
& =K \frac{\partial^{2} q(x, t)}{\partial x^{2}}+\frac{1}{A_{2} \tau_{\mathrm{pr}}(x, t)} S(x, t) .
\end{aligned}
$$

From Eqs. (20) and (15), the equation for supersaturation can be written in the following simple form

$$
\frac{\partial S(x, t)}{\partial t}=K \frac{\partial^{2} S(x, t)}{\partial x^{2}}-\frac{S(x, t)}{\tau_{\mathrm{pr}}(x, t)} .
$$

Eqs. (20) and (20a) show that changes in the microphysical variables are determined by the rate of spatial diffusion (the first term on the right-hand side of these equations) and of evaporation (the second term on the right-hand side).

\section{Analysis of non-dimensional equations}

Spatial diffusion and evaporation depend on many parameters. It is best to start the analysis from the basic equation system presented in a non-dimensional form. A timescale corresponding to the initial phase relaxation time in a cloud volume can be defined as

$\tau_{0}=\frac{\rho_{\mathrm{a}} F}{4 \pi \rho_{\mathrm{w}} A_{2} N_{1} r_{0}}$

and the non-dimensional time is $\tilde{t}=t / \tau_{0}$. Other nondimensional parameters to be used are: the non-dimensional phase relaxation time

$\tilde{\tau}_{\mathrm{pr}}=\tau_{\mathrm{pr}} / \tau_{0}=\frac{N_{1} r_{0}}{N(\widetilde{x}, \widetilde{t}) \bar{r}(\widetilde{x}, \widetilde{t})}$, the normalized liquid water mixing ratio which is equal to the normalized liquid water content

$\widetilde{q}=\frac{q}{q_{1}}$,

the normalized supersaturation

$\widetilde{S}=\frac{S}{A_{2} q_{1}}$,

the non-dimensional conservative function

$\widetilde{\Gamma}=\frac{\Gamma}{A_{2} q_{1}}$,

the normalized square of droplet radius

$\widetilde{\sigma}=\frac{\sigma}{r_{0}^{2}}$,

the normalized droplet concentration

$\widetilde{N}=N / N_{1}$

and the non-dimensional DSD

$\widetilde{g}(\widetilde{\sigma})=\frac{r_{0}^{2}}{N_{1}} g(\sigma)$

with normalization $\widetilde{N}=\int_{0}^{1} \widetilde{g}(\widetilde{\sigma}) d \widetilde{\sigma}$. The definition (Eq. 22g) means that the integral of a non-dimensional initial size distribution over the normalized square radius is equal to unity.

The non-dimensional distance and the non-dimensional time are defined as

$\tilde{x}=x / L ; \quad \tilde{t}=t / \tau_{0}$.

A widely used non-dimensional parameter showing the comparative rates of diffusion and evaporation is the Damkölher number:

$D a=\frac{\tau_{\mathrm{mix}}}{\tau_{0}}=\frac{L^{2}}{K \tau_{0}}$,

where

$\tau_{\text {mix }}=\frac{L^{2}}{K}$

is the characteristic timescale of mixing. Using the nondimensional parameters listed above, Eq. (20) can be rewritten in a non-dimensional form as

$$
\begin{aligned}
\frac{\partial \widetilde{q}(\widetilde{x}, \widetilde{t})}{\partial \widetilde{t}} & =\frac{1}{D a} \frac{\partial^{2} \widetilde{q}(\widetilde{x}, \widetilde{t})}{\partial \widetilde{x}^{2}}+\frac{1}{\widetilde{\tau}_{\mathrm{pr}}(\widetilde{x}, \widetilde{t})}[\widetilde{\Gamma}(\widetilde{x}, \widetilde{t})-\widetilde{q}(\widetilde{x}, \widetilde{t})] \\
& =\frac{1}{D a} \frac{\partial^{2} \widetilde{q}(\widetilde{x}, \widetilde{t})}{\partial \widetilde{x}^{2}}+\frac{1}{\widetilde{\tau}_{\mathrm{pr}}(\widetilde{x}, \widetilde{t})} \widetilde{S}(\widetilde{x}, \widetilde{t})
\end{aligned}
$$

where

$$
\widetilde{q}(\widetilde{x}, \widetilde{t})=\frac{N(\widetilde{x}, \widetilde{t}) \overline{\sigma^{3 / 2}}}{N_{1} r_{0}^{3}}=\int_{0}^{\infty} \widetilde{\sigma}^{3 / 2} \widetilde{g}(\widetilde{x}, \widetilde{t}, \widetilde{\sigma}) \mathrm{d} \widetilde{\sigma} .
$$


The initial conditions and the boundary conditions should be rewritten in a non-dimensional form as well. For instance, the normalized initial condition for the non-dimensional function $\widetilde{q}(\widetilde{x}, 0)$ can be derived from Eqs. (1c) and (22b)

$\widetilde{q}(\tilde{x}, 0)= \begin{cases}1 & \text { if } 0 \leq \tilde{x}<1 / 2 \\ 0 & \text { if } 1 / 2 \leq \tilde{x}<1\end{cases}$

The solution for $\widetilde{\Gamma}(\widetilde{x}, \widetilde{t})$ obtained by a normalization of solution (Eq. 7) is

$$
\begin{aligned}
\widetilde{\Gamma}(\widetilde{x}, \widetilde{t}) & =\frac{1}{2}(1+R)+(1-R) \\
& \sum_{n=1}^{\infty} \frac{\sin (n \pi / 2)}{n \pi / 2} \exp \left(-\frac{n^{2} \pi^{2} \widetilde{t}}{D a}\right) \cos (n \pi \widetilde{x}),
\end{aligned}
$$

where

$R=\frac{S_{2}}{A_{2} q_{1}}$

is a non-dimensional parameter referred to, hereafter, as a potential evaporation parameter (PEP). The PEP is proportional to the ratio of the amount of water vapor that should evaporate in order to saturate the initially droplet-free volume (that is determined by $S_{2}$ ) to the initial available liquid water $q_{1}$ in the cloud volume. The solution of Eq. (28) at $\widetilde{t} \rightarrow \infty$ depends only on parameter $R$.

$\widetilde{\Gamma}(\widetilde{x}, \infty)=\frac{1}{2}(1+R)$

The importance of PEP that determines a possible final state was illustrated in Pt1. PEP is also the sole parameter enabling calculation of the normalized mixing diagram for homogeneous mixing (Pt2). In this study, we consider cases when $R<0$ since $S_{2}<0$, i.e., when droplets can only evaporate in the course of mixing.

The solution of Eq. (25) and the type of mixing depends on the values of two non-dimensional parameters, namely, $D a$ and $R$. Thus, when $R=\frac{S_{2}}{A_{2} q_{1}}<-1, \widetilde{\Gamma}(\widetilde{x}, \infty)<0$. It means that the initially droplet-free volume $V_{2}$ is too dry and all the droplets in the mixing volume evaporate completely. At the final equilibrium state $\mathrm{RH}<100 \%$, i.e., $S(x, \infty)<0$. If $R=\frac{S_{2}}{A_{2} q_{1}}>-1, \widetilde{\Gamma}(\widetilde{x}, \infty)>0$. This means that the mixed volume in the final state contains droplets, i.e., the mixing leads to an increase of the volume with droplets, i.e., the cloud volume. At the final equilibrium state, $\mathrm{RH}=100 \%$ (i.e., $S(x, \infty)=0$ ). The case when $|R|=\left|\frac{S_{2}}{A_{2} q_{1}}\right|=\left|\widetilde{S}_{2}\right| \ll 1$ corresponds to either RH close to $100 \%$ (i.e., $S_{2}$ is close to zero) (this case corresponds to the degenerated case considered in Pt1), and/or to the case when the liquid water mixing ratio in the cloud volume is large. In case $|R| \ll 1$, the second term on the right-hand side of Eq. (25) is much smaller than the first term, and the mixing is driven by turbulent diffusion only.
In case $D a \rightarrow 0$ (often considered as homogeneous mixing), at the beginning of the mixing the diffusion term is much larger than the evaporation term, the second term on the right-hand side of Eq. (25). As mixing proceeds, within a short time period the total homogenization of all the variables in the mixing volume is established and all the spatial gradients become equal to zero. At this time instance, the first term on the right-hand side becomes equal to zero, and the second term on the right-hand side of Eq. (25), describing droplet evaporation, becomes dominant. Thus, the analysis of the Eq. (25) shows that mixing consists of two stages. The first mixing stage is a short stage of inhomogeneous mixing and the longer second stage of homogeneous mixing. The evolution of the microphysical variables during homogeneous mixing is described in detail in Pt2.

$D a \rightarrow \infty$ corresponds to extremely inhomogeneous mixing, according to the classic concept. In this case, the diffusion term is much smaller than the evaporation term, so evaporation takes place under significant spatial gradients of RH. At $D a=\infty$, the adjacent volumes do not mix at all and remain separated. This is equivalent to the existence of two independent adiabatic volumes. Another interpretation of the limiting case $D a=\infty$ is an infinite fast droplet evaporation. Both scenarios at $D a \rightarrow \infty$ indicate simplifications in the definition of the extremely inhomogeneous mixing. At intermediate values of $\mathrm{Da}$, mixing is inhomogeneous, when both turbulent diffusion and evaporation contribute simultaneously to formation of the DSD.

Using Eqs. (14) and normalization (22f), the equations for the non-dimensional size distribution can be written as

$$
\begin{aligned}
\frac{\partial \widetilde{g}(\widetilde{x}, \widetilde{t}, \widetilde{\sigma})}{\partial \widetilde{t}} & =\frac{1}{D a} \frac{\partial^{2} \widetilde{g}(\widetilde{x}, \widetilde{t}, \widetilde{\sigma})}{\partial \widetilde{x}{ }^{2}} \\
& +\frac{2}{3}[\widetilde{\Gamma}(\widetilde{x}, \widetilde{t})-\widetilde{q}(\widetilde{x}, \widetilde{t})] \frac{\partial \widetilde{g}(\widetilde{x}, \widetilde{t}, \widetilde{\sigma})}{\partial \widetilde{\sigma}} .
\end{aligned}
$$

Eq. (31) is solved with the following initial conditions

$$
\widetilde{g}(\widetilde{x}, 0, \widetilde{\sigma})= \begin{cases}\delta(\widetilde{\sigma}-1) & \text { if } 0 \leq \tilde{x}<1 / 2 \\ 0 & \text { if } 1 / 2 \leq \tilde{x} \leq 1\end{cases}
$$

where $\delta(\widetilde{\sigma}-1)$ is a delta function.

Table 1 presents the list of all the non-dimensional variables used in this study and the ranges of their variation. It is shown that six parameters determining the geometrical and microphysical properties of mixing can be reduced to two non-dimensional parameters, which enables a more efficient analysis of mixing. The ranges of parameter variations in Table 1 correspond to the simplifications used in the study (the initial DSD is monodisperse and $\mathrm{RH} \leq 100 \%$ ). 
Table 1. Main parameters of the problem and their non-dimensional forms*.

\begin{tabular}{llll}
\hline Quantity & Symbol & Non-dimensional form & Range of normalized values \\
\hline Time & $t$ & $\tilde{t}=\frac{t}{\tau_{0}}$ & {$[0 \ldots \infty]$} \\
Distance & $x$ & $\widetilde{x}=\frac{x}{L}$ & {$[0 \ldots 1]$} \\
Square of drop radius & $\sigma$ & $\widetilde{\sigma}=\frac{\sigma}{r_{0}^{2}}$ & {$[0 \ldots 1]$} \\
Droplet concentration & $N$ & $\widetilde{N}=\frac{N}{N_{1}}$ & {$[0 \ldots 1]$} \\
Liquid water mixing ratio & $q$ & $\widetilde{q}=\frac{q}{q_{1}}$ & {$[0 \ldots 1]$} \\
Distribution of square of drop radius & $g(\sigma)$ & $\widetilde{g}(\widetilde{\sigma})=\frac{r_{0}^{2}}{N_{1}} g(\sigma)$ & \\
Conservative function & $\Gamma$ & $\widetilde{\Gamma}=\frac{\Gamma}{A_{2} q_{1}}$ & {$[-\infty \ldots 1]$} \\
Supersaturation & $S$ & $\widetilde{S}=\frac{S}{A_{2} q_{1}}$ & {$[-\infty \ldots 0]$} \\
Relaxation time & $\tau_{\mathrm{pr}}$ & $\widetilde{\tau_{\mathrm{pr}}}$ & $\frac{\tau_{0}}{\tau_{0}}$ \\
Damkölher number & $D a$ & $D a=\frac{\tau_{\operatorname{mix}}}{\tau_{0}}=\frac{L^{2}}{K \tau_{0}}$ & {$[0 \ldots \infty]$} \\
Potential evaporation parameter (PEP) & $R$ & $R=\frac{S_{2}}{A_{2} q_{1}}$ & {$[-\infty \ldots 0]$} \\
\hline
\end{tabular}

* All normalized values depend on the initially given values of $L, N_{1}, r_{0}, A_{2}, S_{2}$ and $K$.

\section{Design of simulations}

\subsection{Damköhler number in clouds}

The characteristic mixing time $\tau_{\text {mix }}$ can be evaluated using Eqs. (2) and (24)

$\tau_{\text {mix }}=\frac{1}{C} \varepsilon^{-1 / 3} L^{2 / 3}$.

There is significant uncertainty regarding the evaluation of $\tau_{\text {mix }}$ and Damköhler number, $D a$, in clouds, which is largely related to the choice of coefficient $C$ in expression (33). These values differ in different studies: $C=10$ (Jeffery and Reisner, 2006); $C=1$ (Lehmann et al., 2009) and $C \approx 0.2$ (Monin and Yaglom, 1975) and Boffetta and Sokolov (2002).

According to Lehmann et al. (2009), the values of $D a$ in clouds of different types range from to 0.1 to several hundred. Thus, estimation of $D a$ in clouds may vary within a wide range up to a few orders of magnitude. $D a$ values in stratocumulus clouds can be similar or even higher than those in cumulus clouds, since both $\tau_{\mathrm{mix}}$ and $\tau_{\mathrm{pr}}$ in stratiform clouds are larger than in cumulus clouds.

In our simulations, we compare the evolution of the microphysical parameters within a wide range of $D a$ (from 1 up to 500 ) and of $R$ (from -1.5 up to -0.1 ). $D a=1$ represents the case closest to homogeneous mixing, while $D a=500$ indicates extremely inhomogeneous mixing.

\subsection{Numerical method}

Calculations were performed using MATLAB solver PDEPE. We solve the equation system (Eq. 31) for normalized DSD $\widetilde{g}\left(\widetilde{x}, \widetilde{t}, \widetilde{\sigma}_{j}\right)$ with the initial condition (Eq. 32) and the Neumann boundary conditions

$$
\frac{\partial \widetilde{g}\left(0, \tilde{t}, \widetilde{\sigma}_{j}\right)}{\partial \widetilde{x}}=\frac{\partial \widetilde{g}\left(1, \widetilde{t}, \widetilde{\sigma}_{j}\right)}{\partial \widetilde{x}}=0
$$

where $j=1 . .24$ are the bin numbers on a linear grid of square radii. The number of grid points along the $\tilde{x}$ axis was set equal to 81 .

In calculation of the last term on the right-hand side of Eq. (31), the normalized supersaturation $\widetilde{S}$ was calculated first using the normalized conservative equation

$\widetilde{S}(\widetilde{x}, \widetilde{t})=\widetilde{\Gamma}(\widetilde{x}, \widetilde{t})-\widetilde{q}(\widetilde{x}, \widetilde{t})$,

where $\widetilde{\Gamma}(\widetilde{x}, \widetilde{t})$ is calculated using Eq. (28). Then, this term was formulated using Eq. (9) as

$$
\begin{aligned}
& \frac{2}{3} \widetilde{S}(\widetilde{x}, \widetilde{t}) \frac{\partial \widetilde{g}\left(\widetilde{x}, \tilde{t}, \widetilde{\sigma}_{j}\right)}{\partial \widetilde{\sigma}_{j}} \\
& \quad \approx \frac{\widetilde{g}\left(\widetilde{x}, \widetilde{t}, \widetilde{\sigma}_{j}+\frac{2}{3} \widetilde{S} \Delta \widetilde{t}\right)-\widetilde{g}\left(\widetilde{x}, \widetilde{t}, \widetilde{\sigma}_{j}\right)}{\Delta \widetilde{t}} .
\end{aligned}
$$

Therefore, at each time step, the DSD $\widetilde{g}$ first was shifted to the left of the value $\frac{2}{3} \widetilde{S} \Delta \widetilde{t}$, where $\Delta \widetilde{t}$ is a small time increment chosen so that $\left|\frac{2}{3} \widetilde{S}_{\max } \Delta \widetilde{t}\right| \leq \frac{\Delta \widetilde{\sigma}}{2}$. Next, the shifted DSD was remapped onto the fixed square radius grid $\widetilde{\sigma}_{j}$. We used the remapping method proposed by Kovetz and Olund (1969), which conserves droplet concentration and LWC. After remapping, the differences between the new and old DSDs were recalculated. The new values of LWC were then determined using new values of DSD and Eq. (26). MATLAB utility PDEPE automatically chooses the time step needed to provide stability of calculations.

\section{Results of simulations}

\subsection{Full evaporation case}

First, we consider the case $R=-1.5$, when all the cloud water evaporates completely. This process corresponds to the 

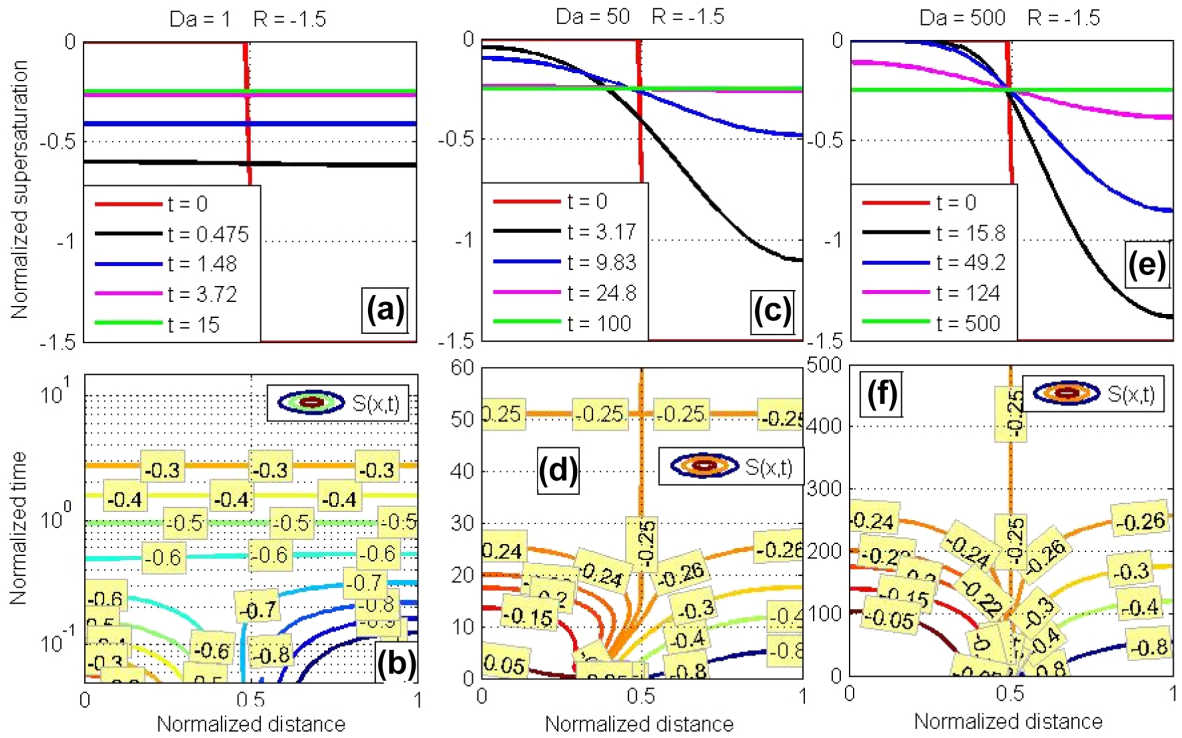

Figure 3. Horizontal dependencies (upper row) and $\tilde{x}-\tilde{t}$ dependencies (lower row) of normalized supersaturation at $D a=1, D a=50$ and $D a=500$ and at $R=-1.5$. Panel (b) is plotted in semi-log scale.
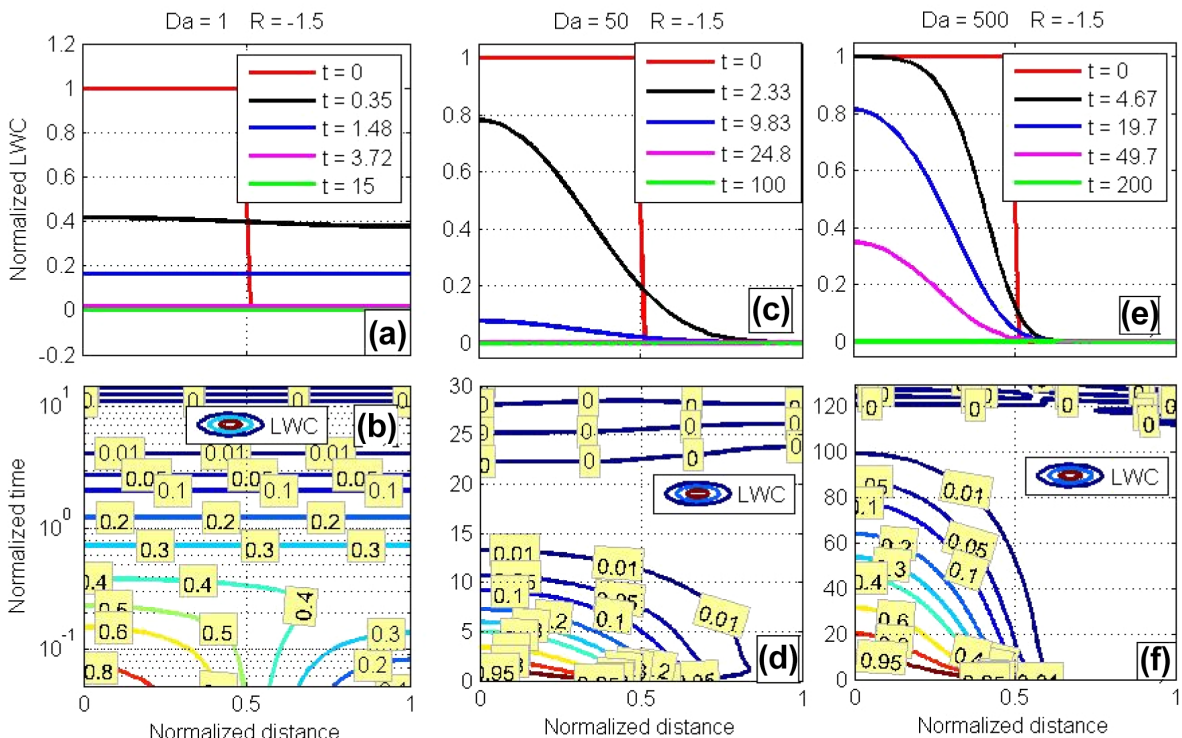

Figure 4. The same as in Fig. 3, but for normalized LWC. Left bottom panel is plotted in semi-log scale.

cloud dissipation caused by mixing with the entrained dry air. At the final state, RH is expected to be uniform and negative over the entire mixing volume.

Figure 3 shows spatial and time changes of $\widetilde{S}$ for $D a=1$, 50 and 500. At the final state for all three cases $\widetilde{S}=-0.25$, which is in agreement with the analytical solution of Eq. (30). The final negative value indicates that all the droplets completely evaporated during mixing. At $D a=1$ (Fig. 3a, b), two stages of supersaturation evolution can be identified. The first short stage with $t<0.4 \tau_{\mathrm{pr}}$ is the period of inhomogeneous mixing, when the gradients of RH persist. By end of the sec- ond stage of about $14 \tau_{\mathrm{pr}}$, the equilibrium state is reached. Thus, at small $D a$ both types of mixing take place. In the cases of $D a=50$ and $D a=500$, the spatial gradients exit during the entire period of mixing until the equilibrium state is reached (approximately $50 \tau_{\mathrm{pr}}$ and $300 \tau_{\mathrm{pr}}$, respectively) (Fig. 3c, d, e, f). Therefore, at these $D a$ mixing is inhomogeneous during entire mixing.

Figure 4 shows spatial changes (upper row) and changes in $\tilde{x}-\tilde{t}$ coordinates (lower row) of normalized LWC for the same case as in Fig. 3. These diagrams demonstrate a significant difference in the evaporation rates at different $D a$ val- 

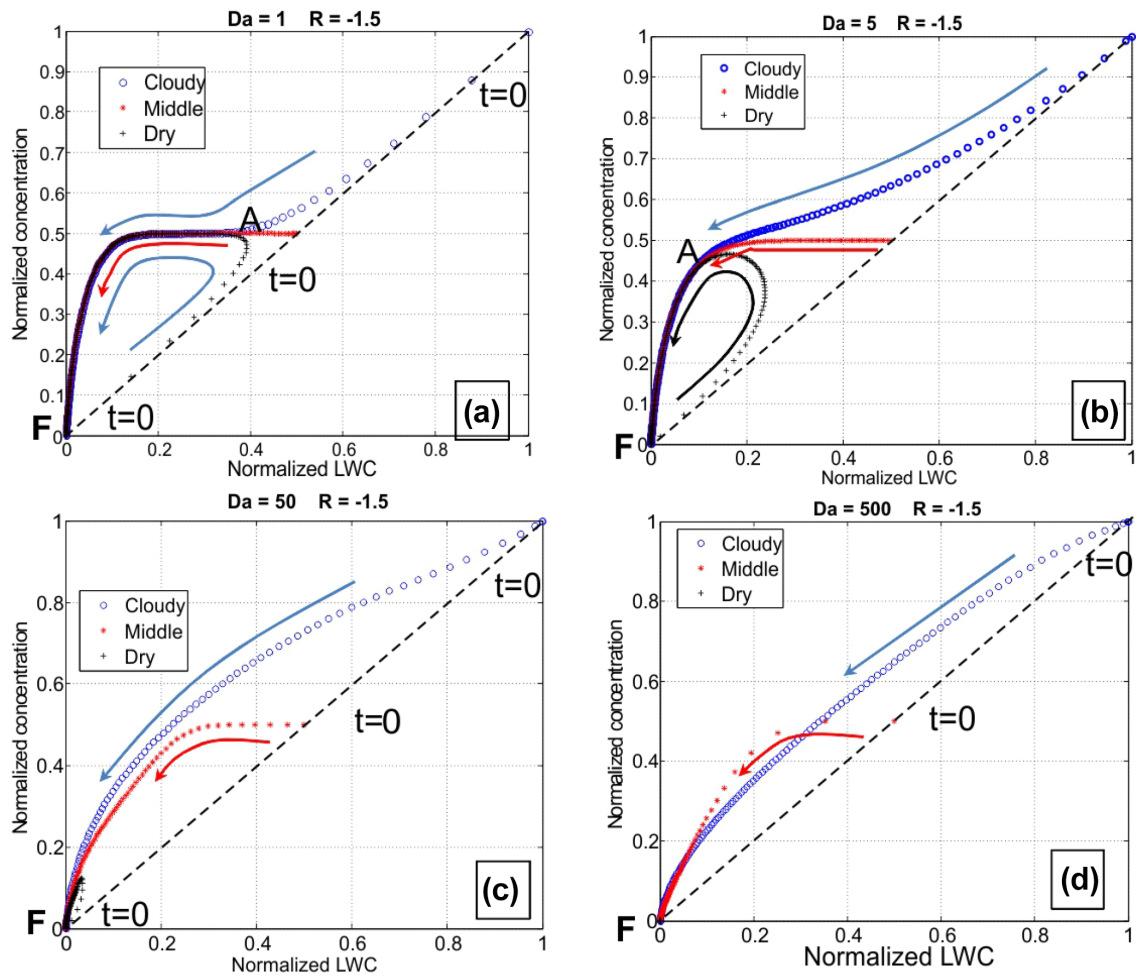

Figure 5. Dependencies of normalized values of droplet concentration on normalized LWC at different $D a$ and $R=-1.5$. Blue symbols mark the center of the cloudy volume $(\tilde{x}=1 / 4)$, red symbols mark the interface between the cloudy volume and the dry volume $(\widetilde{x}=1 / 2)$, and black crosses mark the center of the initially droplet-free volume $(\tilde{x}=3 / 4)$. Symbols are plotted at different time instances. Symbols at $t=0$ show initial values of droplet concentration and LWC at the three values of $\tilde{x}$. Arrows show the direction of movement of the points at the diagram with time. Point "A" marks the beginning of the spatially homogeneous stage, $\tilde{t}=T_{\operatorname{mix}}$. Point "F" marks the final state. The dashed line indicates the relationship between $\widetilde{N}$ and $\widetilde{q}$ in extremely inhomogeneous mixing (according to the classical concept).

ues. Complete evaporation ( $\mathrm{LWC}=0)$ is reached at $D a=1$, 50 and 500 by about 12, 22 and 120 relaxation time periods, respectively.

Analysis of Figs. 3 and 4 allows one to introduce two characteristic time periods: (1) period $T_{\text {mix }}$ during which the spatial gradients of the microphysical parameters persist, and mixing is inhomogeneous, and (2) period $T_{\mathrm{ev}}$ during which droplet evaporation takes place. Both time periods are dimensionless and normalized using $\tau_{0}$. Time period $T_{\mathrm{ev}}$ is equal either to the time of complete droplet evaporation (when $R<-1.0$ ) or to the time period during which the saturation deficit in the mixing volume becomes equal to zero (or close to zero if $R>-1.0$ ), i.e., evaporation is actually terminated. Quantitative evaluations of $T_{\mathrm{mix}}$ and $T_{\mathrm{ev}}$ will be given in Sect. 5.3. At $\widetilde{t}<T_{\mathrm{mix}}$, droplets in the mixing volume experience different saturation deficits. Toward the end of time $T_{\text {mix }}$ the saturation deficit becomes uniform over the entire mixing volume because of mechanic mixing. At $D a=1$, the homogenization of the saturation deficit and all the microphysical variables takes place during a very short time of about $0.5 \tau_{\mathrm{pr}}$, and then the evaporation of droplets is assumed to take place under the same subsaturation conditions, so $T_{\mathrm{mix}} \ll T_{\mathrm{ev}}$.
Figure $4 \mathrm{a}$, b show that at $\widetilde{t} \approx 0.35$, normalized LWC drops down from 1 to 0.4 . Since the average value of the normalized LWC in the mixing volume is equal to 0.5 (see the initial condition in Eq. 27), $20 \%$ of the droplet mass evaporates during this short inhomogeneous period. Thus, despite being quite short, inhomogeneous mixing stage plays an important role even at $D a=1$.

Since at $t=0$ the mixing volume is not spatially homogeneous by definition, there is always a period while spatial inhomogeneity exists. With increasing $D a$, the duration of the inhomogeneous stage increases and the duration of the homogeneous stage decreases. At $D a=500$, homogenization of the saturation deficit requires $250 \tau_{\mathrm{pr}}$, which is twice as long as the time of complete droplet evaporation, i.e., $T_{\mathrm{mix}} \approx 2 T_{\mathrm{ev}}$. This means that at $D a=500$, droplet evaporation takes place in the presence of the spatial gradients of supersaturation. After complete evaporation of droplets, spatial gradients of the water vapor mixing ratios remain. This kind of mixing is regarded as inhomogeneous.

At $D a=50$, the time of complete evaporation is approximately equal to the time of supersaturation homogenization, i.e., $T_{\mathrm{mix}} \approx T_{\mathrm{ev}}$. In this case, as at $D a=500$, the droplets ex- 

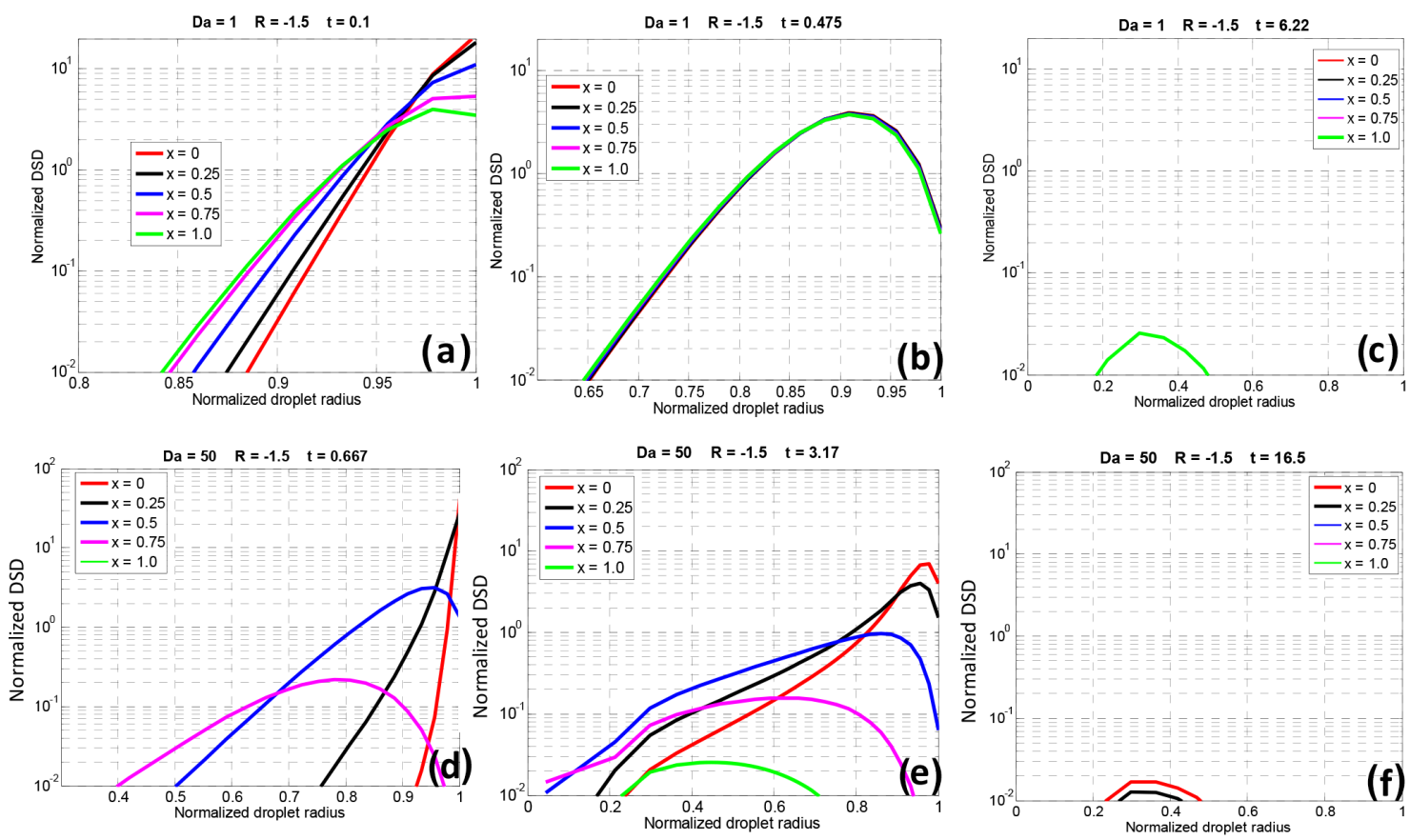

Figure 6. Time evolution of DSD during droplet evaporation at $D a=1$ (upper row) and $D a=50$ (bottom row). In each panel, the normalized DSD are shown at different values of horizontal coordinate $\tilde{x}$. Different panels show DSD at different time instances.

perience different saturation deficit within the mixing volume, so mixing is inhomogeneous at $D a=50$.

The differences in droplet evaporation at different $D a$ can be seen in Fig. 5, showing the relationships between $\widetilde{N}$ and $\widetilde{q}$ plotted with a certain time increment, so that each symbol in the diagrams corresponds to a particular time instance. These symbols form curves. Each panel of Fig. 5 shows three curves corresponding to different $\widetilde{x}$ : the center of the initially cloud volume $(\tilde{x}=1 / 4)$; the center of the mixing volume $(\widetilde{x}=1 / 2)$ and the center of the initially droplet-free volume $(\tilde{x}=3 / 4)$. The directions of the time increase are shown by arrows along the corresponding curves. The initial points of the curves corresponding to $\widetilde{t}=0$ are characterized by values $\widetilde{q}=1$ and $\widetilde{N}=1$ at $\widetilde{x}=1 / 4$, and by values $\widetilde{q}=0$ and $\widetilde{N}=0$ at $\widetilde{x}=3 / 4$.

The behavior of the $\widetilde{N}-\widetilde{q}$ relationship provides important information about mixing process. At $\widetilde{t}<T_{\text {mix }}$, there are spatial gradients of $\widetilde{N}$ and $\widetilde{q}$, i.e., $\widetilde{N}$ and $\widetilde{q}$ are different at different $\widetilde{x}$. This means that the three curves at $\widetilde{t}<T_{\text {mix }}$ do not coincide. At $\tilde{t}>T_{\text {mix }}$, the spatial gradients of $\widetilde{N}$ and $\widetilde{q}$ disappear and the three curves coincide. When the curves do not coincide, mixing is inhomogeneous, and the coincidence of the curves indicates that the mixing becomes homogeneous. In Fig. 5a and b $(D a=1$ and $D a=5$, respectively), the curves coincide at point A corresponding to time $\tilde{t}=T_{\text {mix }}$.

Figure 5a, b show that at $D a=1$ and $D a=5$, mixing consists of two stages: inhomogeneous and homogeneous. The time instance $\widetilde{t}=T_{\mathrm{mix}}$ separates these two stages. In turn, the period of homogeneous mixing (when evaporation is spatially homogeneous) can be separated into two sub-periods. During the first sub-period, droplets evaporate only partially and $\widetilde{q}$ decreases at the same droplet concentration. This subperiod is very pronounced at $D a=1$, when $\widetilde{q}$ decreases from about 0.4 to 0.1 at the unchanged droplet concentration. At the second sub-period, when $\widetilde{q}<0.1$, droplets evaporate completely, beginning with smaller ones, so both the droplet concentration and $\widetilde{q}$ rapidly drop to zero. At $D a=5$ (Fig. 5b), at the stage of homogeneous evaporation (that begins at point "A") the decrease in $\widetilde{q}$ is accompanied by a decrease in $\widetilde{N}$.

At $D a=50$ (Fig. 5c), curves corresponding to different values of $\widetilde{x}$ do not coincide, except at the final point "F", where $\widetilde{N}=0$ and $\widetilde{q}=0$. This means that horizontal gradients exist during the entire mixing process and mixing is inhomogeneous till the final equilibrium state is reached. Droplets penetrating into the initially droplet-free volume begin evaporating, so only a small fraction of droplets reaches the center of the droplet-free volume, as seen in Fig. 5 c, $\widetilde{x}=3 / 4$ (black curve). Accordingly, at $\tilde{x}=3 / 4$ the droplet concentrations and $\widetilde{q}$ reach their maxima (of 0.1 and 0.05 , respectively) and then decrease to zero. At $D a=500$ (Fig. 5d), all the droplets evaporate before reaching the center of the dry volume, indicating an extremely high spatial inhomogeneity of droplet evaporation. Hence, only two curves for $\widetilde{x}=1 / 4$ and $\tilde{x}=1 / 2$ are seen in Fig. $5 \mathrm{~d}$.

Figure 5 also shows that the slopes of the curves describing the $\widetilde{N}-\widetilde{q}$ relationships are different at different values of 

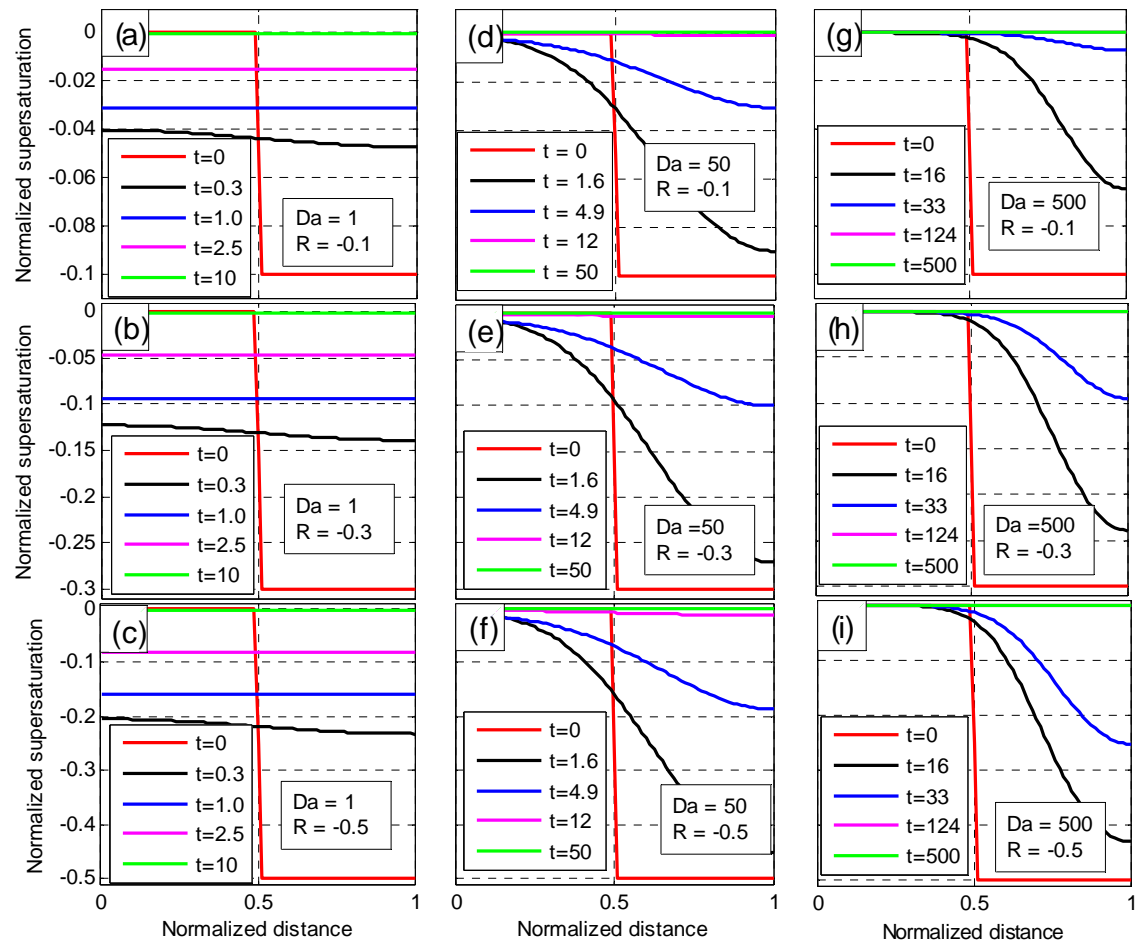

Figure 7. Profiles of normalized supersaturation at different $D a$ and different $R>-1$.

$\widetilde{x}$ and change over time. At large $D a$, the slopes of the curves describing the dependencies $\widetilde{N}-\widetilde{q}$ in the initially cloud volume are close to linear. However, the slope at a high value of $\widetilde{q}$ is still flatter than that at a low value of $\widetilde{q}$. This can be attributed to the fact that when $\widetilde{q}$ is large, it decreases faster than the concentration $\widetilde{N}$ because some fraction of droplets evaporate only partially. At the end of the mixing when $\widetilde{q}$ is small, $\widetilde{N}$ decreases faster than $\widetilde{q}$, because the droplet concentration is determined by the smallest droplets, while $\tilde{q}$ is determined by larger droplets.

As was discussed in Pt1, according to the classical concept of extremely inhomogeneous mixing, the ratio $q / N$ remains constant. For dimensionless $\widetilde{N}$ and $\widetilde{q}$, the scattering points should be aligned along the $1: 1$ line. Therefore, the closeness of particular cases to the classical extremely inhomogeneous mixing can be evaluated by the deviation of the $\widetilde{N}-\widetilde{q}$ curve from the $1: 1$ line. One can see that at $D a=500$ the $\widetilde{N}-\widetilde{q}$ relationship is closer to linear.

Despite the fact that at $R<-1$ all the droplets within the mixing volume evaporate, it is interesting to follow the DSD evolution during this process. Figure 6 shows the time evolution of a normalized DSD at $D a=1$ and $D a=50$. One can see a substantial difference in the DSD evolutions at different $D a$. At $D a=1$, different DSDs are formed very rapidly at different values of $\tilde{x}$ (panel a). The widest DSD occurs at $\tilde{x}=1$, i.e., at the outer boundary of the initially droplet-free volume. This is natural, because the supersaturation deficit is the highest at $\tilde{x}=1$. At $\tilde{t}>T_{\text {mix }} \approx 0.4$, DSD become simi- lar at all values of $\widetilde{x}$ (Fig. 6b). The DSD width continues to increase due to partial droplet evaporation. This time period corresponds to the horizontal segment of the $\widetilde{N}-\widetilde{q}$ relationship in Fig. 5a. Figure 6c shows the DSD at the stage when a decrease in LWC is accompanied by a decrease in number droplet concentration. The corresponding point in the $\widetilde{N}-\widetilde{q}$ diagram at this time instance is quite close to the point " $F$ " at which $\widetilde{N}=0$ and $\widetilde{q}=0$.

At $D a=50, \mathrm{DSD}$ are different at different $\tilde{x}$ during the entire period of mixing. While DSD at $\tilde{x}>0.5$ are wide and droplet evaporation is accompanied by a shift of DSD maximum to smaller droplet radii (this feature is typically attributed to homogeneous mixing), the DSD maximum at $\tilde{x}<$ 0.5 (the initially cloud volume) shifts toward smaller radii only slightly until $\tilde{t}=3.17$ (Fig. 6e). Further droplet evaporation either leads to a complete evaporation (at $\widetilde{x} \geq 0.5$ ) or shifts the DSDs to smaller droplet sizes (panel f). The maximum droplet concentration takes place at $\tilde{x}=0$. Figure 6 shows that DSD shapes evolve substantially over time, although the final state is characterized by complete droplet evaporation.

\subsection{Partial evaporation case}

\subsubsection{Evolution of the microphysical parameters at different values of $D a$ and $R$}

Here we consider the process of mixing at $R>-1$, i.e., when not all the droplets evaporate completely. Figure 7 

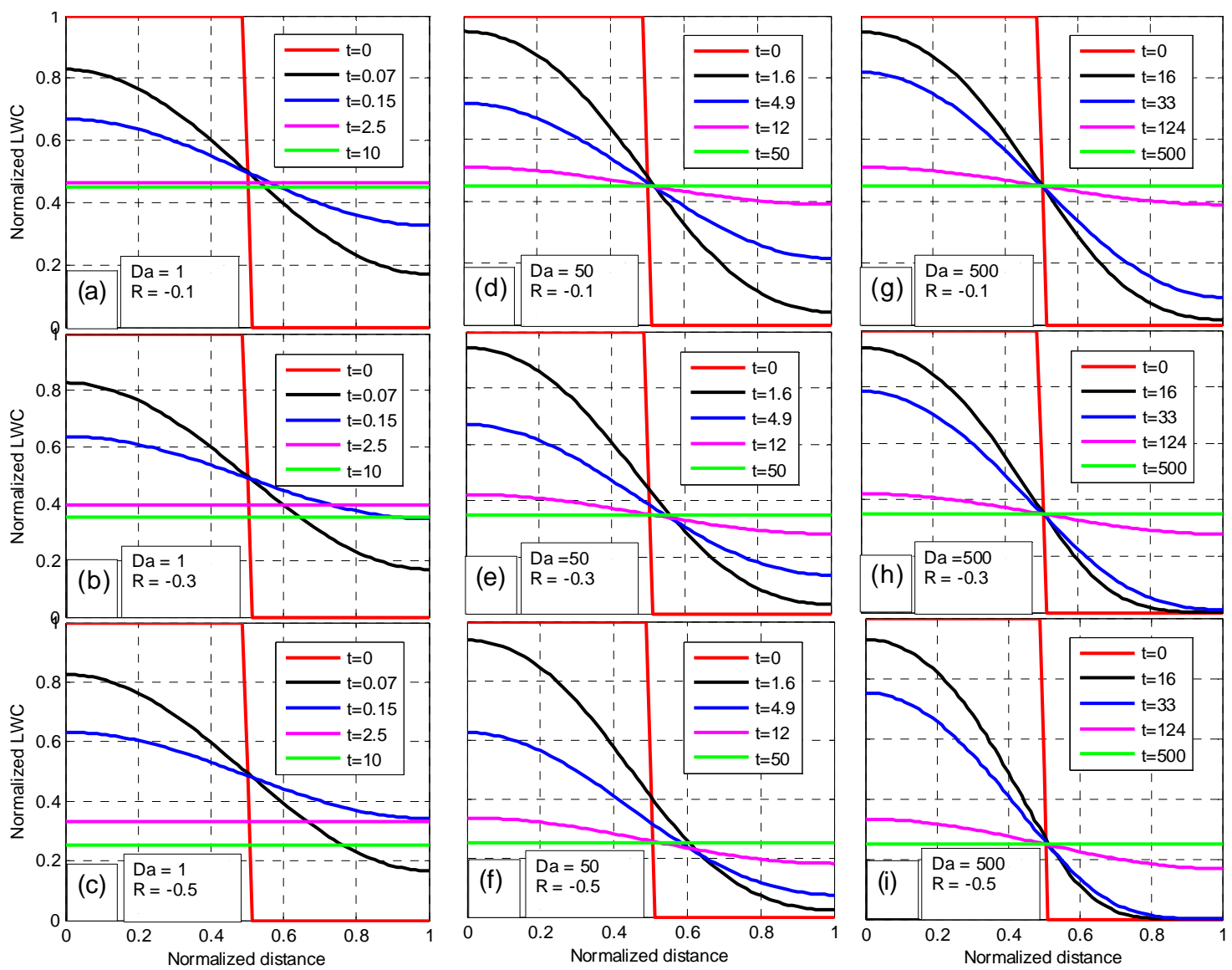

Figure 8. Profiles of normalized LWC at different $D a$ and at different $R>-1$.

shows the horizontal profiles of a normalized supersaturation at different $D a$ and $R$. One can see that in all cases, the final state occurs when the equilibrium supersaturation $\widetilde{S}=0$ $(\mathrm{RH}=100 \%)$. However, this final value is reached quite differently depending on $D a$. At $D a=1$, rapid mixing leads to the formation of spatially homogeneous humidity and supersaturation during a time period of a fraction of $\tau_{\mathrm{pr}}$. Then, supersaturation within the mixing volume grows by evaporation of droplets, which are uniformly distributed over the entire mixing volume. This process of homogeneous mixing was analyzed in detail in Pt2.

At $D a=500$, changes in supersaturation take place largely within the initially droplet-free volume. $\mathrm{RH}$ in the initially cloud volume undergoes only small changes. This process agrees well with the classical concept of extremely inhomogeneous mixing. However, a strong gradient of supersaturation remains within the initially drop-free volume for a long time (tens of $\tau_{\mathrm{pr}}$ ). At $D a=50$, the situation is intermediate. Mixing is intensive enough to decrease RH in the initially cloud volume, but spatially uniform RH is established within about $5-10 \tau_{\mathrm{pr}}$, increasing with an increase in $|R|$. After this time instance, mixing takes place according to the homogeneous scenario.
Figure 8 shows the horizontal profiles of normalized LWC at different $D a$ and $R$. At the same $R$, the final equilibrium values of LWC are identical, as follows from Eq. (30); LWC decreases with an increase in $|R|$. At any $D a$, the decrease in the LWC in the cloud volume is caused largely by diffusion of droplets from the cloud volume into the initially dropletfree volume.

At $D a=500$, evaporation in the cloud volume is small because $\widetilde{S}$ in these volumes is high during mixing (Fig. 7). At $D a=1$, the process of spatial homogenization takes place during fractions of $\tau_{\mathrm{pr}}$, i.e., $T_{\mathrm{mix}}<1$. Then, during a relatively lengthy period of $10 \tau_{\mathrm{pr}}$, evaporation decreases LWC over the entire mixing volume, which is characteristic of homogeneous mixing. At $D a=50$, spatial homogenization takes place during about $T_{\operatorname{mix}} \approx 15$. This is a slightly shorter time than it takes to establish the final equilibrium stage $T_{\text {tot }}$. Different $D a$ cases reach equilibrium at different times. The process of reaching a final uniform LWC lasts for $100 \tau_{\mathrm{pr}}$ at $D a=500$ and for about $\tau_{\mathrm{pr}}$ at $D a=1$.

Figure 9 shows the profiles of the normalized droplet concentrations at different $D a$ and $R$. In contrast to LWC, the final concentration depends both on $D a$ and $R$. Hence, profiles at different $D a$ can have different shapes at the same value of 

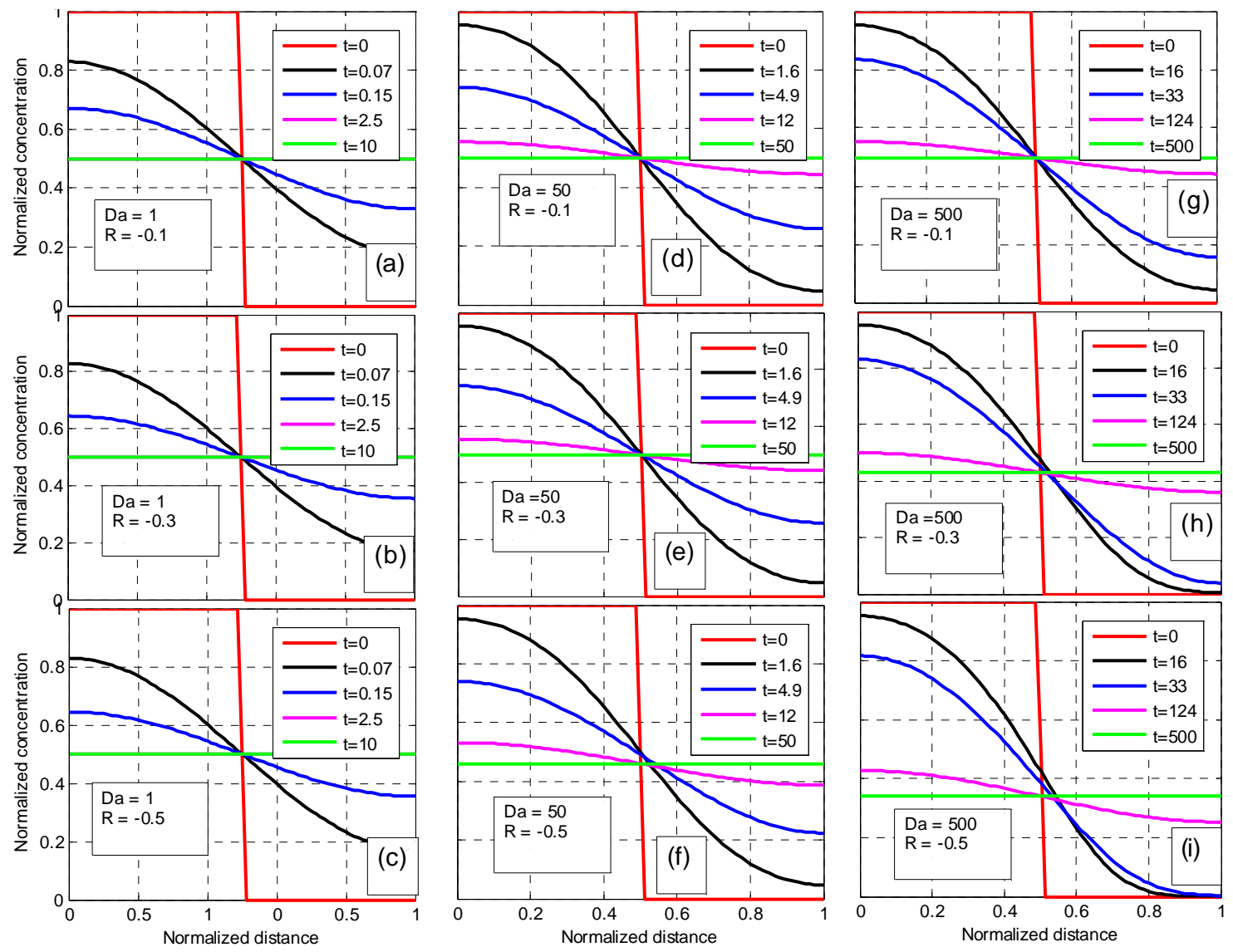

Figure 9. Profiles of normalized droplet concentration at different $D a$ and at different $R>-1$.

$R$. At $R=-0.1$ (which corresponds to high RH in the initially dry volume) none of the droplets evaporate, so the final normalized droplet concentration is equal to $\widetilde{N}=1 / 2$. This means that all the droplets in the initially cloud volume are now uniformly distributed between both mixing volumes. At larger $|R|$, i.e., at lower RH in an initially droplet-free volume, some droplets evaporate completely. The final concentration decreases with an increase in $D a$.

The physical interpretation of this dependence is clear. At low $D a$, fast mixing leads to formation of a uniform $\mathrm{RH}$ throughout the entire mixing volume, and this affects all the droplets. At high $D a, \mathrm{RH}$ in the initially droplet-free volume remains low for a long time, and droplets that penetrate can evaporate. Therefore, the fraction of completely evaporated droplets increases with $D a$ : at $R=-0.1$ there are no completely evaporated droplets at any $D a$. At $R=-0.3$ a decrease in the droplet concentration takes place only at $D a=500$, and at $R=-0.5$ the droplet concentration decreases already at $D a \geq 50$.

The comparative contributions of different factors in establishing the final states of mixing are well seen in Fig. 10 presenting the relationships between normalized concentration and normalized LWC at three values of $\widetilde{x}: 1 / 4$ (center of the cloudy volume), $1 / 2$ and $3 / 4$ (center of the initially dry volume) at $R=-0.5$ and different values of $D a$. Figure 10 is analogous to Fig. 5, but plotted for $R>-1$.

At $D a=1$ the mixing is very fast, which leads to a rapid decrease in LWC and in the droplet concentration in the initially cloud volume and to an increase of these quantities in the initially droplet-free volume. As a result of the rapid mixing and homogenization, all the curves coincide at point " $\mathrm{A}$ " (left panel). After this time instance, spatial homogeneous evaporation takes place. Since at $D a=1$ only partial, but not total, droplet evaporation occurs, the droplet concentration remains unchanged even while LWC decreases. At $D a=50$ and $D a=500$, the three curves coincide at the final point "F" only. At $D a=500$, the relationship between the droplet concentration and the mass becomes more linear (blue curve). The linear dependence is consistent with the concept of extremely inhomogeneous mixing (see Pt1). Considerations regarding the closeness of the $\widetilde{N}-\widetilde{q}$ relationship to the line $1: 1$ as a measure of inhomogeneity of mixing made at $R<-1$ are also valid for $R>-1$. 

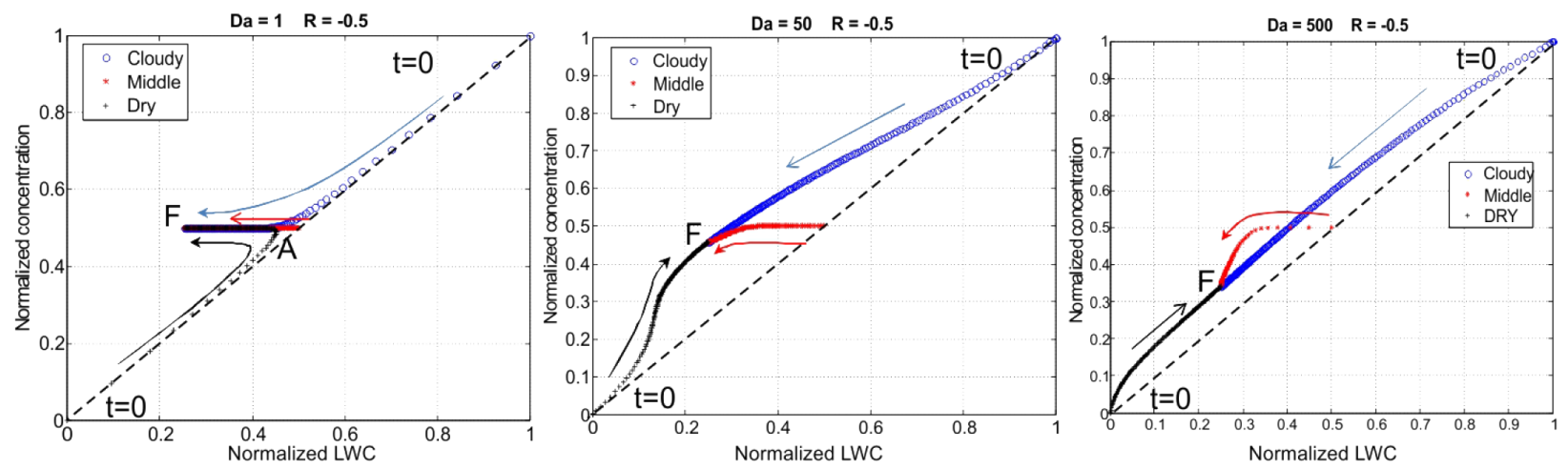

Figure 10. Dependencies of normalized values of droplet concentration on normalized LWC at different $D a$ and at $R=-0.5$. Blue circles mark the center of the cloudy volume $(\tilde{x}=1 / 4)$, red symbols mark the initial interface $(\tilde{x}=1 / 2)$ and black crosses mark the center of the initially dry volume $(\tilde{x}=3 / 4)$. Arrows show the direction of movement of the points with time. Point "F" marks the final stationary state of the system. The dashed line indicates the relationship between $\widetilde{N}$ and $\widetilde{q}$ in extremely inhomogeneous mixing (according to the classical concept).
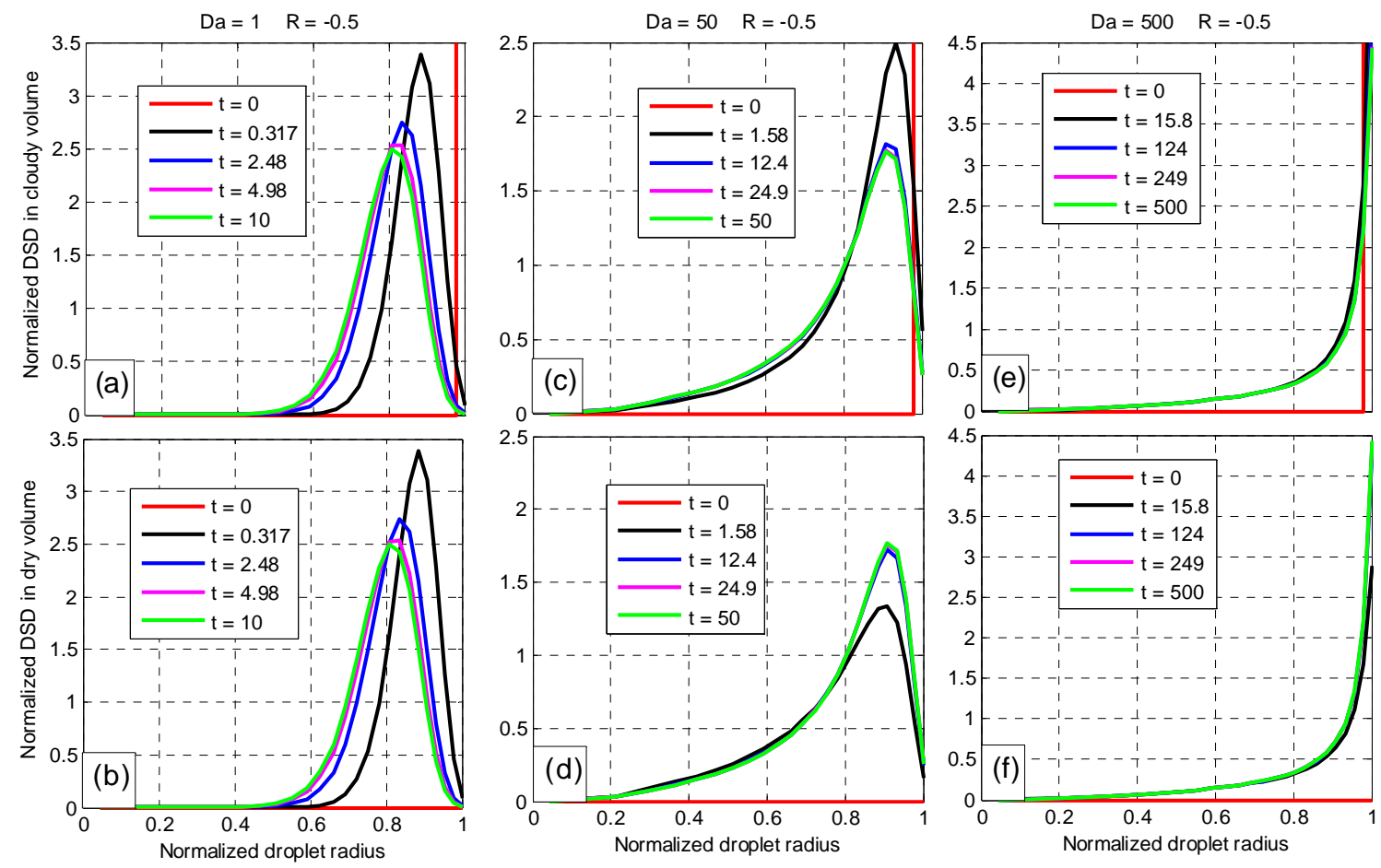

Figure 11. Examples of DSD evolution in the initially cloudy volume $(\tilde{x}=1 / 4)$ (upper row) and in the initially dry volume $(\tilde{x}=3 / 4)($ lower row) at $R=-0.5$ and at different values of $D a$.

\subsubsection{Evolution of DSDs and the DSD parameters}

Figure 11 presents examples of the DSD evolution at the center of the initially cloud volume $(\widetilde{x}=1 / 4)$ (upper row) and of the initially droplet-free volume $(\widetilde{x}=3 / 4)$ at $R=-0.5$ and different values of $D a$. Several specific features of the DSD are notable. As a result of the rapid mixing at $D a=1$ (left column), DSD become similar in both volumes already at $t=0.317 \tau_{\mathrm{pr}}$ (black lines). Further evolution is similar in both volumes and is characterized by broadening of the DSD and its shifting toward smaller droplet sizes. This shift means a decrease in the mass at constant droplet concentration, which is typical of homogeneous mixing.

The initially monodisperse DSDs become polydisperse. The mechanism of the DSD broadening at $D a=1$ is illustrated in Fig. 12, showing the DSD at the earlier, inhomogeneous stage at different $\widetilde{x}$. One can see that within very short periods when the spatial gradient of saturation deficit exists, 

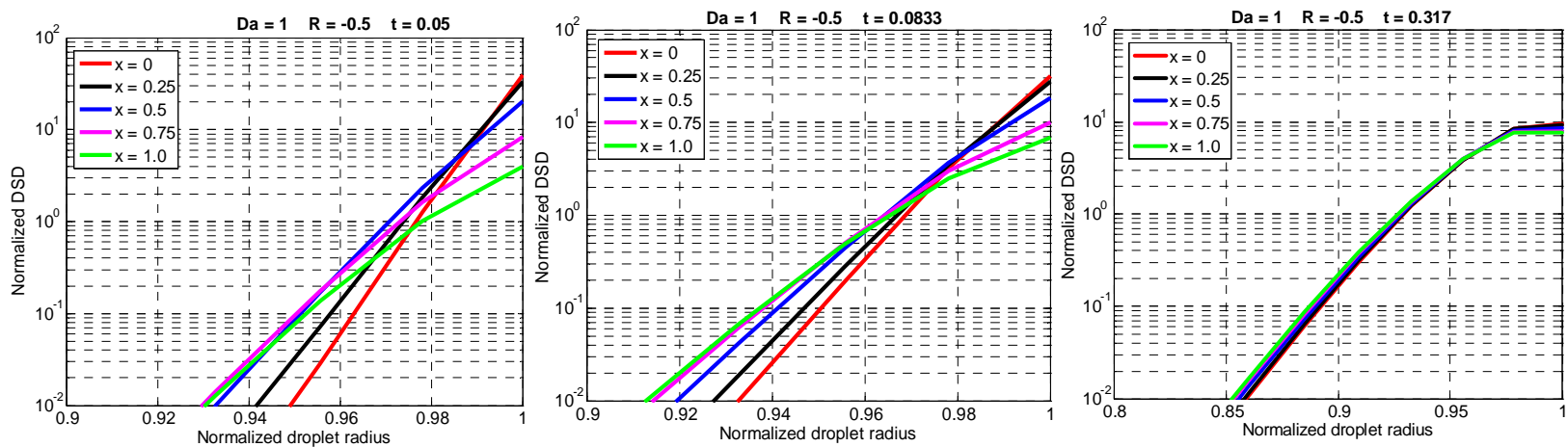

Figure 12. DSD at different $\tilde{x}$ at the beginning of the mixing process for $D a=1$ and $R=-0.5$.
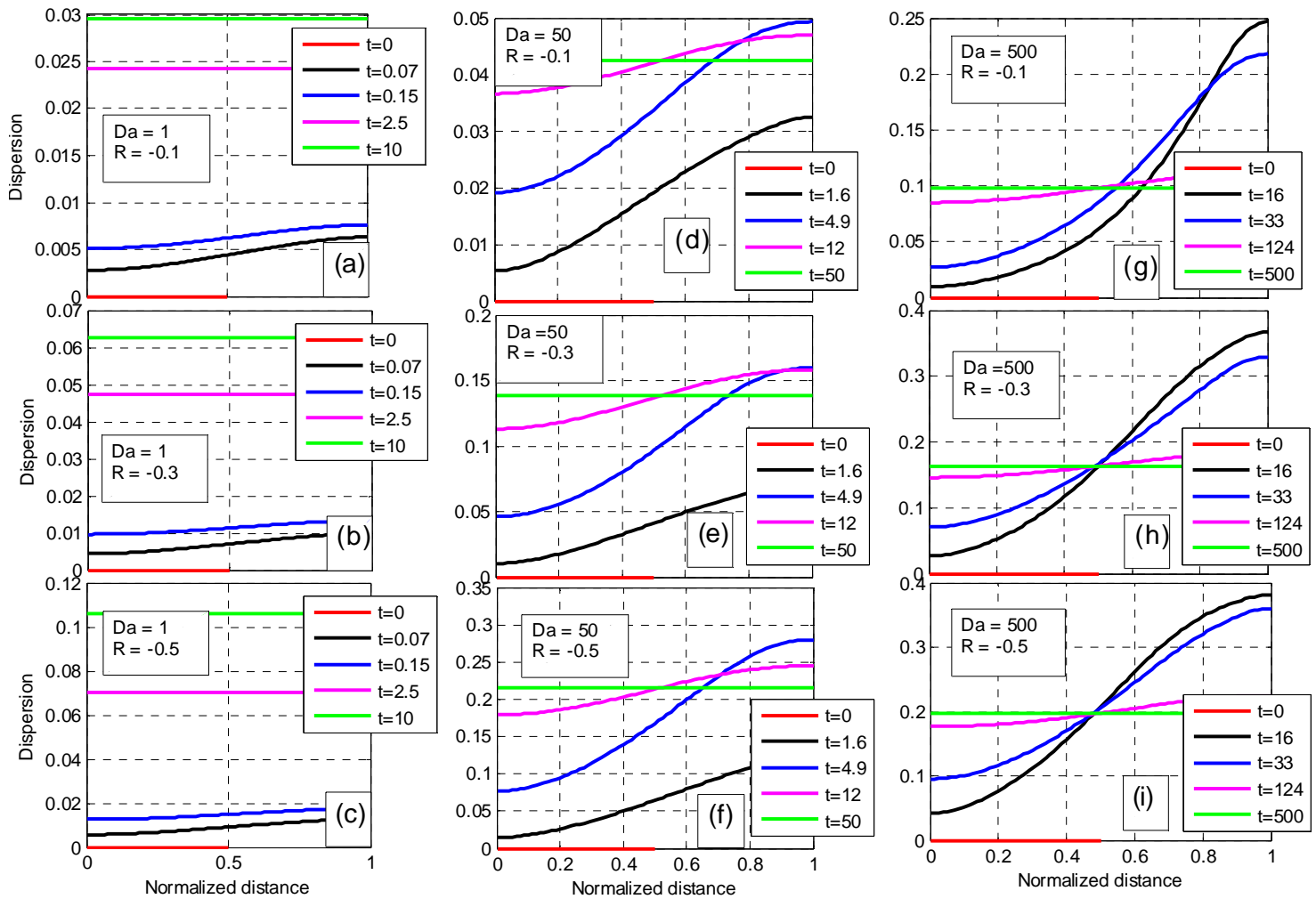

Figure 13. Spatial dependencies of the relative DSD dispersion at different time instances and at different values of $D a$ and different $R>-1$.

droplets entering the initially droplet-free volume partially evaporate, reaching their minimal size at $\tilde{x}=1$. In this way, a polydisperse DSD forms. As the mixing proceeds, DSD become spatially homogenized, as seen in the right panel of Fig. 12.

At $D a=50$ and $D a=500$, the DSD shapes substantially differ from those at $D a=1$. There are two main differences: the peak of the distribution shifts only slightly (at $D a=50$ ) or does not shift at all (at $D a=500$ ). At the same time, the DSD develops a long tail of small droplets. Since the mixing rate at these values of $D a$ is slow, droplets penetrating deeper into the initially dry volume remain there for a long time and get smaller. As a result, at moderate and large $D a$, a polydisperse DSDs form with droplet sizes ranging from zero to 1. Formation of a long tail of small droplets in case of inhomogeneous mixing was simulated in direct numerical simulation (DNS) by Kumar et al. (2012), as well as by means of "the explicit-mixing parcel model" (EMPM) (Krueger et al., 1997; Su et al., 1998; Schlüter, 2006).

Figure 13 shows the spatial dependencies of the DSD dispersion (ratio of DSD r.m.s. width and the mean radius) at different time instances and different values of $D a$ and $R$. One can see that the dispersion increases with an increase in $D a$ and in $|R|$. This behavior can be accounted for by the 

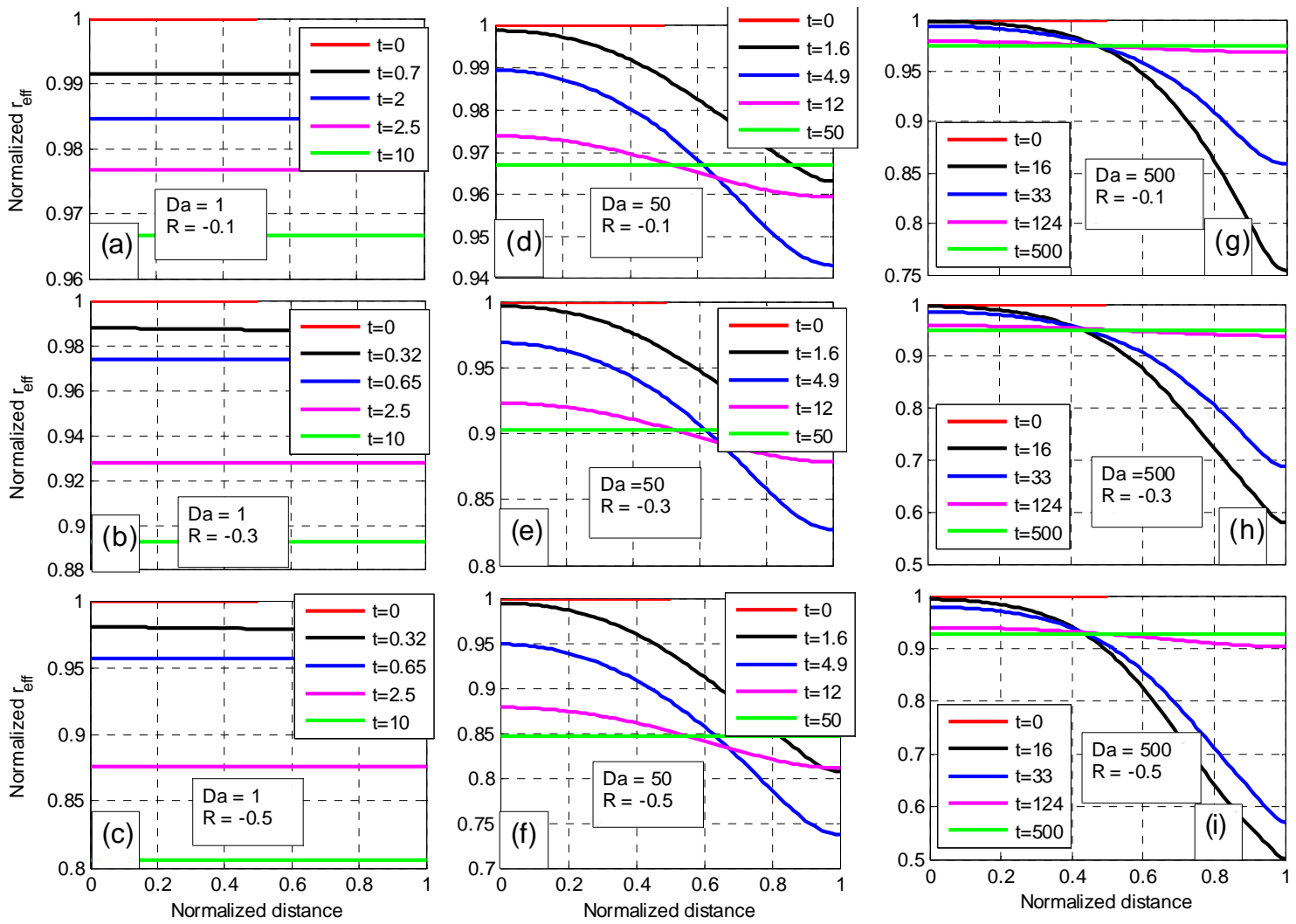

Figure 14. Spatial dependencies of effective radius at different time instances and at different values of $D a$ and different $R>-1$.

fact that the DSD broadening toward smallest droplet size increases with the increase in $D a$ and in $|R|$. The DSD dispersion increases with time and with an increase in $\widetilde{x}$, i.e., further into the initially droplet-free volume. At the same time, spatial homogenization takes place, so at the final state at $R=-0.5$ the DSD dispersion reaches 0.11 at $D a=1$ and about 0.2 at $D a=50$ and $D a=500$.

Observed DSD dispersion in different clouds typically ranges from 0.1 to 0.4 (Khain et al., 2000; Martin et al., 1994; Prabha et al., 2012) and can be caused by the following factors: in-cloud nucleation (e.g., Khain et al., 2000; Pinsky and Khain, 2002), spatial averaging along aircraft traverses (Korolev, 1995) and non-symmetry in droplet nucleation/denucleation (Korolev, 1995). As seen in Fig. 13, this dispersion may be also caused by mixing at cloud edges at moderate and large $D a$. Hence, inhomogeneous mixing leads to DSD broadening.

The effective radius, $r_{\text {eff }}$, is an important DSD characteristic. According to the classical concept, $r_{\text {eff }}$ remains unchanged during extremely inhomogeneous mixing, whereas decreases during homogeneous mixing. Figure 14 shows spatial dependencies of $r_{\text {eff }}$ at different time instances and different values of $D a$ and $R$. At $R=-0.1$ (high RH in the surrounding volume) $r_{\text {eff }}$ is similar for all values of $D a$. So, at high $R$ (i.e., close to zero), the behavior of $r_{\text {eff }}$ does not allow to distinguish between mixing types.

At a given $R$, the final $r_{\text {eff }}$ increases with increasing $D a$. For instance, at $R=-0.5, r_{\text {eff }}$ at the final state differs from the initial $r_{\text {eff }}$ value by less than $6 \%$ at $D a=500$, while at $D a=1 r_{\text {eff }}$ decreases by $20 \%$. At moderate and high $D a$, large gradients of $r_{\text {eff }}$ exist during the mixing process. However, the gradient is high only in the initially droplet-free volume where $r_{\text {eff }}$ decreases significantly due to the intense evaporation of droplets. Besides, $r_{\text {eff }}$ grows very rapidly in the initially droplet free volume, so at high $D a$ during most of the mixing time $r_{\text {eff }}$ within the mixing volume becomes close to the initial $r_{\text {eff }}$ value in the cloudy volume.

\subsection{Delimitation between mixing types}

Typically, the $D a$ value is used as a criterion for delimitation between mixing types. $D a=1$ is usually used as a boundary value separating homogeneous and inhomogeneous mixing. As shown in Sect. 4, mixing always starts as inhomogeneous. In the course of mixing, the initial spatial gradients decrease and the air volumes either become identical or remain different. In the former case, the second mixing stage is homogeneous. If inhomogeneity persists until the equilibrium state is established, mixing remains inhomogeneous during the entire period. Both mixing stages can be characterized 
by duration, change in the droplet concentrations or LWCs, and other quantitative characteristics. These characteristics are functions of two non-dimensional parameters $R$ and $D a$, which can be calculated and used for delimitation between mixing types. Since mixing between volumes may turn from inhomogeneous into homogeneous before reaching the equilibrium state, it is necessary to use some quantitative criteria to delimit mixing types. Below, delimitation is performed for $R>-1$ which corresponds to partial evaporation of droplets by the end of mixing.

\subsubsection{Characteristic time periods of mixing}

Three characteristic time periods of mixing are distinguished: (a) mixing period $T_{\mathrm{mix}}$, during which spatial gradients are smoothing (may be also called the homogenization period); (b) period $T_{\mathrm{ev}}$ during which $S<0$ and droplets evaporate until saturation is reached and (c) the total mixing period $T_{\text {tot }}$ that lasts until the final equilibrium stage is reached. In our analysis, all three periods are assumed to be dimensionless quantities.

We use solution (28) for conservative function $\widetilde{\Gamma}(\widetilde{x}, \widetilde{t})$ to define quantitatively time period $T_{\text {mix }}$. The deviation of the solution from its final value $\Delta \widetilde{\Gamma}=\widetilde{\Gamma}(\widetilde{x}, \widetilde{t})-\widetilde{\Gamma}(\widetilde{x}, \infty)$ at $\widetilde{t} \rightarrow$ $\infty$ can be approximately estimated using the first term of the series expansion as

$$
\begin{aligned}
|\Delta \widetilde{\Gamma}|_{\max } & \approx\left|(1-R) \frac{\sin (\pi / 2)}{\pi / 2} \exp \left(-\frac{\pi^{2} \widetilde{t}}{D a}\right) \cos (\pi \widetilde{x})\right|_{\max } \\
& =(1-R) \frac{2}{\pi} \exp \left(-\frac{\pi^{2} \widetilde{t}}{D a}\right)
\end{aligned}
$$

From Eq. (37) the estimation of $T_{\text {mix }}$ can be written as

$$
T_{\operatorname{mix}}=-\frac{D a}{\pi^{2}} \ln \left[\frac{\pi}{2(1-R)}|\Delta \widetilde{\Gamma}|_{\max }\right] .
$$

Suppose the value of the maximum deviation is $|\Delta \widetilde{\Gamma}|_{\max }=$ 0.02 . This is a small value compared to the initial leap of function $\widetilde{\Gamma}$, which is equal to $1-R$. At $|\Delta \widetilde{\Gamma}|_{\max }=0.02$ the duration of the non-homogeneous stage is evaluated as

$$
T_{\text {mix }}=-\frac{D a}{\pi^{2}} \ln \left[\frac{0.01 \pi}{1-R}\right] \text {. }
$$

Several studies evaluate the evaporation time for droplets of a particular size using the equation for diffusion growth (e.g., Lehmann et al., 2009). In our study, the evaporation time duration $T_{\mathrm{ev}}$ is defined as the period during which the maximum deviation of supersaturation from zero exceeds the small value chosen as $|\Delta \widetilde{S}|_{\max }=0.02$ :

$$
\left|\widetilde{S}\left(\widetilde{x}, T_{\mathrm{ev}}\right)\right| \leq|\Delta \widetilde{S}|_{\max }=0.02 \text {. }
$$

Although criterion (Eq. 39) is rather subjective, it has an advantage over the criterion used by Lehmann et al. (2009), as

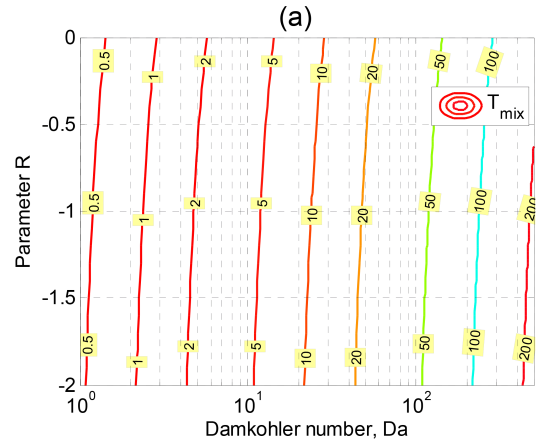

(b)

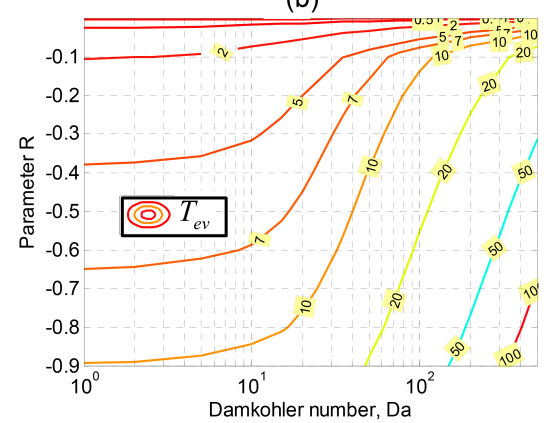

(c)

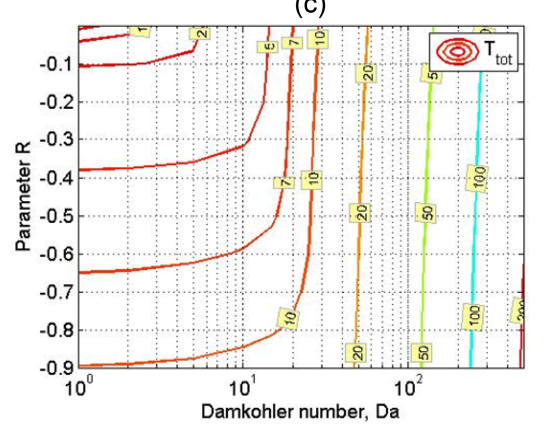

Figure 15. Contours of normalized mixing duration times on $D a-R$ plane. (a) Mixing time $T_{\mathrm{mix}}$, (b) evaporation time $T_{\mathrm{ev}}$, and (c) the total duration mixing time $T_{\text {tot }}$.

Eq. (32) characterizes evaporation of the droplet population taking into account the simultaneous increase in supersaturation, but not of individual droplets of particular size at constant $S$ as in Lehmann et al. (2009).

At the end of the mixing, both the thermodynamic equilibrium and the diffusion equilibrium are reached. Accordingly, the total time of mixing $T_{\text {tot }}$ is evaluated as the maximum of the two time periods needed to achieve equilibrium $T_{\text {tot }}=\max \left\{T_{\mathrm{mix}}, T_{\mathrm{ev}}\right\}$. All the three characteristic time periods are normalized on the phase relaxation time, and, therefore, depend on the two non-dimensional parameters $R$ and $D a$. The contours of the characteristic time durations $T_{\text {mix }}$, $T_{\text {ev }}$ and $T_{\text {tot }}$ in the $D a-R$ diagrams are shown in Fig. 15.

As follows from Eq. (38b), $T_{\text {mix }}$ is proportional to $D a$. The dependence of $T_{\text {mix }}$ on $R$ is not very strong, so $T_{\text {mix }}$ slightly decreases with increasing $R$. This can be attributed to the fact that the lower the $R$, the smaller the initial inhomogeneity 


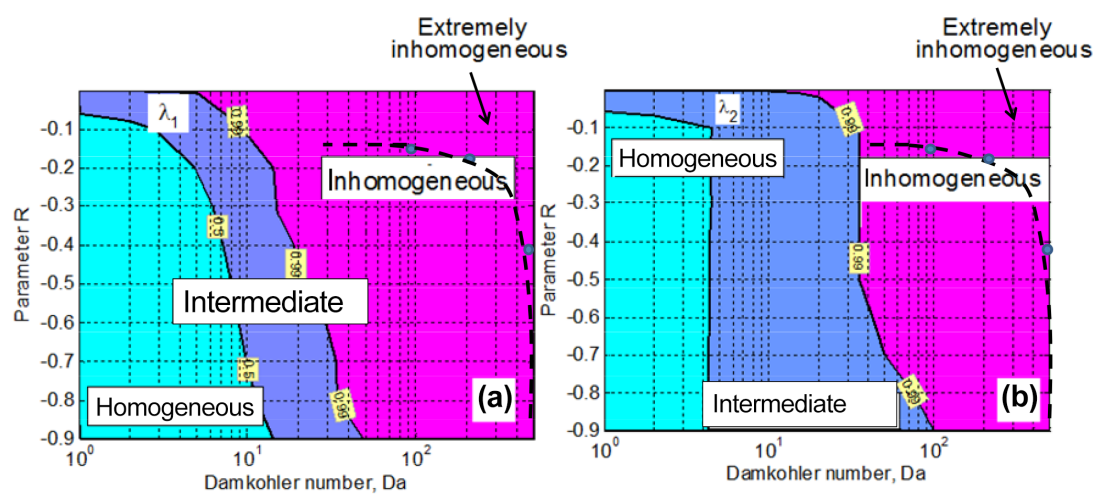

Figure 16. (a) The boundaries between mixing types on the $D a-R$ plane designed according to criteria $\lambda_{1}=\frac{T_{\operatorname{mix}}}{T_{\mathrm{tot}}}$; (b) the boundaries between mixing types on the $D a-R$ plane designed according to criterion $\lambda_{2}=\frac{2\left\langle\widetilde{q}\left(T_{\text {mix }}\right)\right\rangle-1}{R}$ (Eq. 41). Dashed lines indicate the line corresponding to $2 \%$ deviation from the initial mean volume radius.

of function $\widetilde{\Gamma}$ and the shorter the time to align this inhomogeneity is. At small $D a$ (high rate of homogenization of the volume), $T_{\mathrm{ev}}$ depends largely on $R$. At large $D a, T_{\mathrm{ev}}$ depends substantially on $D a$, since the evaporation rate depends on the number of droplets that diffuse to drier parts of the mixing volume. A comparison of Fig. 15c with Fig. 15a and b shows that at small $D a$, time $T_{\text {tot }}$ is determined by $T_{\mathrm{ev}}$, while at large $D a, T_{\text {tot }}$ is determined by $T_{\text {mix }}$.

\subsubsection{Determination of boundaries between the mixing types on the $R-D a$ plane}

Several criteria can be proposed for delimitation between mixing types. We consider these criteria for $R>-1$. As discussed above, mixing always starts as inhomogeneous and late either become homogeneous or remains inhomogeneous till the final equilibrium state is established. At small $D a$, the homogenization takes place during $T_{\text {mix }}<T_{\text {tot }}$. The value of time fraction $\lambda_{1}$ of the inhomogeneous stage can serve as a criterion for definition of homogeneous mixing. This formula for the fraction can be written as

$\lambda_{1}=\frac{T_{\text {mix }}}{T_{\text {tot }}}$.

The case $\lambda_{1} \leq 0.5$, most of the time the mixing takes place according the homogeneous scenario and such a regime is reasonable to regard as homogeneous mixing. If $\lambda_{1}(R, D a)$ changes within the range of $0.5<\lambda_{1} \leq 1$, mixing appears to be intermediate. The criterion (Eq. 40) depends on the non-dimensional parameters $R$ and $D a$. Figure 16a shows the boundaries separating mixing types on the $D a-R$ plane. These boundaries separate all plane into several zones. At very small $R$, the duration of the phase transition is negligibly small. According to criterion (Eq. 40), in this case mixing should be considered inhomogeneous, irrespective of the $D a$ value.

Another criterion of delimitation between mixing types can be determined from a comparison of LWC variation rates due to different mechanisms. The mean normalized LWC (which is equal to the mean normalized liquid water mixing ratio) can be written as integral $\langle\widetilde{q}(\widetilde{t})\rangle=\int_{0}^{1} \widetilde{q}(\widetilde{x}, \widetilde{t}) \mathrm{d} \widetilde{x}$. The initial mean LWC is equal to $\langle\widetilde{q}(t=0)\rangle=\frac{1}{2}$. The final equilibrium LWC is equal to $\langle\widetilde{q}(t=\infty)\rangle=\frac{1}{2}(1+R)$ (Eq. 30). The total amount of liquid water that evaporates in the course of mixing can be quantified by the difference between these two values $\langle\widetilde{q}(t=0)\rangle-\left\langle\widetilde{q}(t=\infty\rangle=-\frac{1}{2} R\right.$. The amount of liquid water evaporated in the course of the first inhomogeneous mixing stage is calculated by the equation $\langle\widetilde{q}(t=0)\rangle-\left\langle\widetilde{q}\left(T_{\text {mix }}\right)\right\rangle=\frac{1}{2}-\left\langle\widetilde{q}\left(T_{\text {mix }}\right)\right\rangle$. Hence, parameter $\lambda_{2}$ which is a ratio of

$\lambda_{2}=\frac{\langle\widetilde{q}(t=0)\rangle-\left\langle\widetilde{q}\left(T_{\text {mix }}\right)\right\rangle}{\langle\widetilde{q}(t=0)\rangle-\langle\widetilde{q}(t=\infty)\rangle}=\frac{2\left\langle\widetilde{q}\left(T_{\text {mix }}\right)\right\rangle-1}{R}$

can serve as another possible criterion for delimitation between mixing types. This ratio characterizes the fraction of liquid water that evaporates at the initial inhomogeneous stage. Condition $\lambda_{2}<0.5$ in this case corresponds to homogeneous mixing, while condition $0.5 \leq \lambda_{2}<1$ corresponds to intermediate mixing. We regard the case $\lambda_{2}=1$ as inhomogeneous mixing. Certainly, criterion $\lambda_{2}$ depends on the non-dimensional parameters $R$ and $D a$. Figure 16b illustrates delimitation between mixing types on the $D a-R$ plane according to criterion $\lambda_{2}$.

Comparison of Fig. 16a and b shows that both criteria lead to nearly similar separation of the $D a-R$ plane into three zones corresponding to homogeneous, intermediate and inhomogeneous mixing. At the same time, the boundaries separating these zones are different depending on the delimitation criterion used. Nevertheless, it can be concluded that mixing can be considered homogeneous at $D a$ below 4-10 and $R<-0.1$ and inhomogeneous at $D a$ exceeding several tens.

Terms "inhomogeneous mixing" (Burnet and Brenguier, 2007) and "extremely inhomogeneous mixing" (Lehmann et 


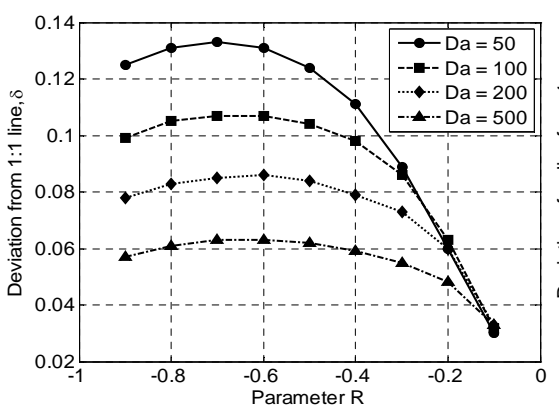

(a)

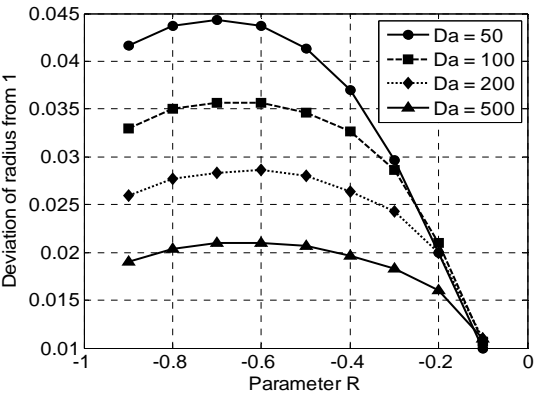

(b)

Figure 17. (a) Dependencies of the r.m.s. distance of the $\widetilde{N}-\widetilde{q}$ relationship curve from straight line $1: 1$ suggested by classical concept of extremely inhomogeneous mixing. The dependencies are plotted for different values of $D a$ and $R$. (b) The same as to the left panel but for r.m.s. deviations of the mean volume radius curve from that initial constant value assumed in the classical concept.

al., 2009; Gerber et al., 2008; Pt1) are used to denote the mixing regime when the relationship between the normalized values $\widetilde{N}$ and $\widetilde{q}$ is represented by a straight $1: 1$ line, which is equivalent to the constant mean volume radius (in some studies, the effective radius is used instead of the mean volume radius. According to the definition used in the present study, extremely inhomogeneous mixing is the limiting case of inhomogeneous mixing when $D a \rightarrow \infty$. Despite the fact that the extremely inhomogeneous mixing is only an idealization our approach allows to determine to what extent mixing can be considered to be close to this limiting case. The measure of inhomogeneity of mixing is the closeness of the $\widetilde{N}-\widetilde{q}$ relationship to the $1: 1$ straight line (see discussion above related to Figs. 5 and 10).

Figure 17 a shows rms distance between the $\widetilde{N}-\widetilde{q}$ relationship and the $1: 1$ straight line, depending on $D a$ and $R$. These dependences were calculated using the set of points $\widetilde{N}_{i}, \widetilde{q}_{i}$ uniformly distributed over spatial interval [0 1$]$ and time interval $\left[0 T_{\text {tot }}\right]$. The equation for estimation is $\delta=$ $\sqrt{\frac{1}{2 M} \sum_{i=1}^{M}\left(\tilde{N}_{i}-\widetilde{q}_{i}\right)^{2}}$, where $M$ is the total number of points. This distance corresponds to r.m.s. deviation of the normalized mean volume radius from 1 . The dependences of the last deviation on $D a$ and $R$ and estimated as $\delta / 3$ are shown in Fig. 17b. This estimation is based on the fact that the total mass of droplets is proportional to the cube of the mean volume radius. As expected, the distance decreases with increasing $D a$. At large $R$, all the curves coincide indicating a degenerative case when type of mixing becomes indistinguishable.

We choose the value $\delta / 3$ equal to 0.02 to determine the boundary of the extremely inhomogeneous mixing zone. The value of 0.02 corresponds to droplet radii deviation of a few tenths of a micron, which is so low that in in situ measurements this case would always be attributed to extremely inhomogeneous mixing. In Fig. 16 this boundary is marked by the broken line. The boundary shows that the mixing at $D a$ exceeding several hundred can be attributed to the extremely inhomogeneous. Between the boundary separating inhomogeneous mixing from the intermediate one and the boundary separated inhomogeneous mixing from extremely inhomogeneous there exists a wide zone of inhomogeneous mixing where the mean volume (or the effective) radius may drop by $10 \%$ and more (Fig. 14), and where the DSD dispersion is substantial and the tail of small droplets is long enough (Fig. 11). Mixing diagrams currently used for analysis of observed data $(N-q$ dependences in the final equilibrium state of mixing) do not contain this zone which, therefore, has remained unrecognized and uninvestigated.

\section{Summary and conclusions}

In this study, inhomogeneous turbulent mixing is investigated using a simple 1-D model of mixing between a saturated cloud volume and an undersaturated droplet-free volume. The mixing is simulated by solving a diffusion-evaporation equation written in the non-dimensional form. For simplicity, the initial volumes of cloudy and droplet-free air were assumed to be equal, and the initial DSD in the cloudy volume was assumed monodisperse.

Analysis of the diffusion-evaporation equation shows that the time-dependent process of mixing and the final equilibrium state depend on two non-dimensional parameters. The first parameter $R$, referred in this paper as potential evaporation parameter (PEP) is proportional to the ratio between the saturation deficit in the initially droplet-free volume and the initial liquid water content in the cloudy volume. At $R<-1$, the final state is characterized by complete droplet evaporation and a spatially homogeneous saturation deficit, which indicates dissipation of the cloudy volume. At $R>-1$, the final state is characterized by existence of droplets and zero saturation deficit $(\mathrm{RH}=100 \%)$. In this case, the cloud volume expands after mixing with the entrained air. At small values of $|R|$ (e.g., when $\mathrm{RH}$ in the entrained volume is close to $100 \%$ ), the effect of droplet evaporation on microphysics is small, and, formally, this kind of mixing should 
be regarded as extremely inhomogeneous. Strictly speaking, this is a degenerate case, when homogeneous and inhomogeneous mixing cannot be distinguished (see also Pt1). At $R=0$, the droplet population turns into a passive admixture and its turbulent diffusion will be the same at different thermodynamic parameters.

The second parameter is the Damkölher number $(D a)$, which is the ratio between the characteristic mixing time and the phase relaxation time. This parameter compares the rates of spatial diffusion and evaporation. Parameter $D a$ (Eq. 23) logically appears in the non-dimensional form of the diffusion-evaporation equation showing that $D a$ is the ratio of the mixing time defined as $\tau_{\text {mix }}=\frac{L^{2}}{K}$, to the initial drop relaxation time. The expression for this non-dimensional parameter clearly shows that since we consider an ensemble of evaporating droplets, the drop relaxation time evaluated just before the mixing is the characteristic timescale of inhomogeneous mixing process. In several studies (e.g., Baker and Latham, 1979; Burnet and Brenguier, 2007; Andejchuk et al., 2009) a question was raised as to which timescale should be used in formulation of the Damkölher number: the time of an individual droplet evaporation at constant saturation deficit, or the phase relaxation time. This study, as well Pt2 show that the phase relaxation time is the answer. The mixing time is introduced via the turbulent diffusion coefficient which is a natural measure characterizing the diffusion rate and, in particular, determines the propagation rate of the fronts in the fields of droplet concentration and other microphysical parameters. The turbulent diffusion coefficient is widely used to describe mixing in cloud models at resolved scales.

The analysis was performed within a wide range of $D a$ (from 1 to 500) and of $R$ (from -1.5 to -0.1 ). The final LWC and the humidity in the mixing volume are determined by the mass conservation and do not depend on $D a$ (see also Pt1 and Pt2). At the same time, the droplet concentration, as well as the shape of DSD and their parameters strongly depend on $D a$.

It is shown that the mixing of air volumes with initially different thermodynamical and microphysical parameters consists of two stages characterized by two time periods: the time during which microphysical characteristics become uniform over the total mixing volume $T_{\text {mix }}$, and the time during which zero saturation deficit is reached (at $R>-1$ ), $T_{\mathrm{ev}}$. At $\widetilde{t}<T_{\text {mix }}$, the spatial gradients of the microphysical values remain and the mixing regime can be regarded as inhomogeneous. At $\tilde{t}>T_{\mathrm{mix}}$, droplet evaporation, if it occurs at all, takes place within a spatially homogeneous medium, so all the droplets in the mixing volume experience equal saturation deficit. This regime can be regarded as homogeneous. It is shown, therefore, that at small $D a$ mixing between two volumes that starts as inhomogeneous can become homogeneous towards the end of mixing.

This finding allows to delimit between mixing types. We presented two quantitative criteria on the $D a-R$ plane that allow to delimit three mixing regimes: homogeneous, intermediate and inhomogeneous. These criteria are based on comparison of the characteristic duration mixing and the evaporation rates. According to the criteria, at $D a$ below about 5 , mixing can be regarded as homogeneous, i.e., the main microphysical changes take place during the homogeneous stage. At $5<D a<50$, the changes in the microphysical parameters are more significant at the inhomogeneous stage than at the homogeneous stage. In this case, the mixing can be regarded as intermediate. Finally, at $D a$ exceeding several tens, the spatial microphysical gradients remain until the final equilibrium stage is reached. In this case, the mixing can be regarded as inhomogeneous. At $D a$ exceeding a few hundred the deviations from predictions based on the classical concept of extremely inhomogeneous become relatively small, which justifies regarding this mixing to as extremely inhomogeneous.

On the whole, the results of the present study are in line with the classic concepts defining homogeneous and inhomogeneous mixing types. However, several important points emerge from our work show serious limitations of classical concepts. A comparison of the classical concepts and the present study is presented in Table 2. Analysis of Table 2 shows the following.

a. In contrast to many studies that analyze only the hypothetical final (equilibrium) state of mixing (Burnet and Brenguier, 2007; Gerber et al., 2008; Morrison and Grabowski, 2008; Hill et al., 2009), we consider the entire time-dependent processes of mixing and evaporation. At moderate and high $D a$, the mixing can last several minutes. In in situ observations, we see mostly nonequilibrium stages which may account for a rather wide scattering of mixing diagrams even at the same values of $D a$ (e.g., Lehmann et al., 2009).

Note that time-dependent mixing was also considered in several studies (e.g., Baker et al., 1980; Baker and Latham, 1982; Jeffery and Reisner, 2006; Krueger et al., 1997; Kumar et al., 2012) using different approaches and numerical models. These studies, however, do not contain analysis on non-dimensional diffusionevaporation equation.

b. It is also shown in the study that the slopes of the $\widetilde{N}-\widetilde{q}$ relationship (between the normalized droplet concentration and LWC) tends to the $1: 1$ line with increasing $D a$. The closeness can be considered as a measure of extremely inhomogeneous mixing in terms of the classical concept (see Pt1). It has been found that the slope of the $\widetilde{N}-\widetilde{q}$ relationship depends on the LWC and, accordingly, on time. At large LWC, $\widetilde{q}$ changes with time faster than $\widetilde{N}$, while at low LWC the concentration changes faster. Although mixing types are usually separated into homogeneous and extremely inhomogeneous, we have shown that there are wide ranges of $D a$ and $R$ at which 
Table 2. Comparison of analysis based on the classic concepts of mixing and the results of the present study.

\begin{tabular}{|c|c|}
\hline Classical concept & The present study \\
\hline $\begin{array}{l}\text { Only the final equilibrium state is typically analyzed; } \\
\text { results of in situ observations are interpreted assuming } \\
\text { the equilibrium state. }\end{array}$ & $\begin{array}{l}\text { The mixing period can last several minutes and more. } \\
\text { The microphysical structure of the mixing volumes dur- } \\
\text { ing this period can differ substantially from that at the } \\
\text { final state }\end{array}$ \\
\hline $\begin{array}{l}\text { Types of mixing are separated into homogeneous and } \\
\text { extremely inhomogeneous. }\end{array}$ & $\begin{array}{l}\text { There are the wide ranges of } D a \text { and } R \text { values, at which } \\
\text { mixing can be regarded as intermediate or inhomoge- } \\
\text { neous (but not extremely inhomogeneous). }\end{array}$ \\
\hline Mixing can start as purely homogeneous & Any mixing starts with the inhomogeneous stage \\
\hline $\begin{array}{l}\text { Homogeneous mixing leads to a DSD shift to small } \\
\text { droplet sizes }\end{array}$ & $\begin{array}{l}\text { Homogeneous mixing does not always lead to the DSD } \\
\text { shift to small droplet sizes (Pt2). The shift depends on } \\
\text { the DSD shape. }\end{array}$ \\
\hline $\begin{array}{l}\text { Mixing can be analyzed within the framework of a } \\
\text { monodisperse DSD }\end{array}$ & Mixing always leads to formation of polydisperse DSD \\
\hline $\begin{array}{l}\text { In the course of homogeneous mixing, droplet concen- } \\
\text { tration remains constant }\end{array}$ & $\begin{array}{l}\text { In the course of homogeneous mixing, droplet concen- } \\
\text { tration does not always remain constant }(\mathrm{Pt} 2)\end{array}$ \\
\hline $\begin{array}{l}\text { Extremely inhomogeneous mixing does not change the } \\
\text { DSD shape }\end{array}$ & $\begin{array}{l}\text { Inhomogeneous mixing (including extremely inhomo- } \\
\text { geneous) leads to broadening of the DSD towards small } \\
\text { sizes }\end{array}$ \\
\hline $\begin{array}{l}\text { In the course of inhomogeneous mixing, the effective } \\
\text { radius remains constant }\end{array}$ & $\begin{array}{l}\text { The effective radius varies only slightly ( } 5-20 \%) \text { in the } \\
\text { initially cloud volume. The effective radius rapidly in- } \\
\text { creases in the initially droplet-free volume, approaching } \\
\text { the value of effective radius in the cloud volume. With } \\
\text { increasing } D a \text {, the difference between the values of the } \\
\text { effective radius in the initially cloud volume and that at } \\
\text { the final state decreases in agreement with the classic } \\
\text { concept. }\end{array}$ \\
\hline
\end{tabular}

mixing should be considered intermediate or inhomogeneous, but not extremely inhomogeneous. Within these ranges the effective radius can change by more than $10-15 \%$. Standard mixing diagrams do not include this range that, to our knowledge, has never been investigated despite the fact that multiple in situ measurements indicate its existence (e.g., Lu et al., 2014).

c. Many studies assume the existence of pure homogeneous mixing during which the initially monodisperse DSD remains monodisperse. Our study shows that at the very beginning, mixing is always inhomogeneous. This inhomogeneous stage leads to the formation of a polydisperse DSD that broadens in the course of droplet evaporation. Hence, even at $D a=1$ the initially monodisperse spectrum becomes polydisperse.

d. It is shown that at small $D a$, mixing includes both inhomogeneous and homogeneous stages, which means that type of mixing can change during the mixing process.

e. The classical concept assumes that the effective radius always decreases during homogeneous mixing. Assum- ing an initially monodisperse DSD, we have found this conclusion largely valid, with the exception of small $R$. At the same time, it was shown in Pt2 that during homogeneous mixing, the effective radius can decrease, remain constant or increase depending of the initial DSD shape. Thus, a decrease in the effective radius during mixing cannot always be considered an indication of homogeneous mixing. Similarly, the invariability of the effective radius during mixing in the process cannot always be considered an indication of extremely inhomogeneous mixing.

f. It is generally assumed that during homogeneous mixing droplet concentration remains unchanged. In the present study, as well as in Pt2, it is shown that since mixing leads to a polydisperse DSD, the smallest droplets may completely evaporate. At $R<-1$, the DSD becomes very wide and all the droplets, the smallest ones first, evaporate.

g. It is generally assumed that inhomogeneous mixing does not alter DSD shape, but only decreases droplet 
concentration. The present study showed that inhomogeneous mixing significantly changes the DSD shape. DSD were found to be quite different in different regions of mixing volumes. The main feature is the DSD broadening toward small droplet size, so the relative dispersion grows up to $0.2-0.3$. These values are quite close to those observed in atmospheric clouds (Khain et al., 2000). Elongated tails of small droplets during mixing were simulated by Schlüter (2006) who described turbulent diffusion following Krueger et al. (1997) and Su et al. (1998) as well as Kumar et al. (2012) using DNS. We see that formation of a polydisperse DSD is a natural result of inhomogeneous mixing and, therefore, inhomogeneous mixing is an important mechanism of DSD broadening. A significant impact of mixing on DSD shape was found identified in multiple studies, beginning with Warner (1973).

h. The effective radius has been assumed to remain constant during extremely inhomogeneous mixing. Our results indicate that, indeed, at the final equilibrium stage at comparatively high RH the effective radius is close to that in the initially cloudy volume (especially at high $D a$ ). At the same time, we found that the effective radius varies in size and is smaller in the initially droplet-free volumes.

The results obtained in parts Pt 1 and Pt2, and especially in the current study $(\mathrm{Pt} 3)$ dedicated to analysis of turbulent mixing mechanisms in clouds determine the directions for future work. Since the widely used mixing diagrams show only a hypothetical equilibrium state, but not the instantaneous state of mixing that likely correspond to transition periods, the efficiency of the standard mixing diagrams is questionable. Moreover, the standard diagrams miss a very important mixing regime, namely, inhomogeneous mixing that occurs between two limiting cases of homogeneous and extremely inhomogeneous mixing (Fig. 16).

We believe that the results obtained will help to improve understanding and interpretation of mixing process both in in situ measurements and modeling. The approach allows us to investigate the relationship between the main microphysical parameters typical of inhomogeneous mixing, that differ from those in the limiting cases of extremely inhomogeneous mixing. In addition, utilization of polydisperse DSD when solving diffusion-evaporation equation allows to investigate the role of the initial DSD shape in mixing. In situ measurements (e.g., Burnet and Brenguier, 2007; Gerber et al., 2008; Lehmann et al., 2009) and numerical models (Magaritz-Ronen et al., 2016) show a wide scattering of data on the scattering diagrams. We expect that the location of various points on the diagrams (e.g., $r_{\mathrm{v}}^{3}$ vs. dilution rates) depends on the shape of the initial DSDs and characterizes the stage of mixing. The method applied in the study allows for the investigation of evolution of DSD moments over space and time.

Recently, there has been vigorous discussions concerning the possible existence of a high humidity layer near cloud edges that might affect mixing of cloud with its surroundings (Gerber et al., 2008; Lehmann et al., 2009). In our opinion, this layer does exist and forms as a result of turbulent mixing of cloud with surrounding dry air, accompanied by complete droplet evaporation. The approach developed in the present paper allows one to analyze formation of such humid layers.

We believe that the results obtained in this study will foster the development of physically grounded parameterization of mixing in cloud models.

\section{Data availability}

Numerical codes of the model are available upon request. 


\section{Appendix A: List of symbols}

Table A1. List of symbols.

\begin{tabular}{|c|c|c|}
\hline Symbol & Description & Units \\
\hline$A_{2}$ & $\frac{1}{q_{\mathrm{v}}}+\frac{L_{\mathrm{w}}^{2}}{c_{p} R_{\mathrm{V}} T^{2}}$, coefficient & nd \\
\hline$a_{0}, a_{n}$ & the Fourier series coefficients & nd \\
\hline C & the Richardson's law constant & nd \\
\hline$c_{p}$ & specific heat capacity of moist air at constant pressure & $\mathrm{J} \mathrm{kg}^{-1} \mathrm{~K}^{-1}$ \\
\hline 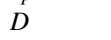 & coefficient of water vapor diffusion in the air & $\mathrm{m}^{2} \mathrm{~s}^{-1}$ \\
\hline$D a$ & the Damkölher number & nd \\
\hline$e$ & water vapor pressure & $\mathrm{Nm}^{-2}$ \\
\hline$e_{s}$ & saturation vapor pressure above a flat water surface & $\mathrm{Nm}^{-2}$ \\
\hline$F$ & $F=\frac{\rho_{\mathrm{W}} L_{\mathrm{W}}^{2}}{k_{\mathrm{a}} R_{\mathrm{y}} T^{2}}+\frac{\rho_{\mathrm{w}} R_{\mathrm{v}} T}{e_{s}(T) D}$, coefficient & $\mathrm{m}^{-2} \mathrm{~s}$ \\
\hline$f(r)$ & $\begin{array}{l}K_{\mathrm{a}} K_{\mathrm{V}} I^{2} \\
\text { droplet size distribution }\end{array}$ & $\mathrm{m}^{-4}$ \\
\hline$g(\sigma)$ & distribution of square radius & $\mathrm{m}^{-5}$ \\
\hline$\widetilde{g}(\widetilde{\sigma})$ & normalized distribution of square radius & nd \\
\hline$k_{\mathrm{a}}$ & coefficient of air heat conductivity & $\mathrm{J}^{-1} \mathrm{~s}^{-1} \mathrm{~K}^{-1}$ \\
\hline$K$ & turbulent diffusion coefficient & $\mathrm{m}^{2} \mathrm{~s}^{-1}$ \\
\hline$L$ & characteristic spatial scale of mixing & $\mathrm{m}$ \\
\hline$L_{\mathrm{W}}$ & latent heat for liquid water & $\mathrm{J} \mathrm{kg}^{-1}$ \\
\hline$m_{\alpha}$ & moment of DSD of order $\alpha$ & $m^{-3}$ \\
\hline$N$ & droplet concentration & \\
\hline$\widetilde{N}$ & normalized droplet concentration & nd \\
\hline$N_{1}$ & Initial droplet concentration in a cloud volume & $m^{-3}$ \\
\hline$p$ & pressure of moist air & $\mathrm{Nm}^{-2}$ \\
\hline$q$ & liquid water mixing ratio & $\mathrm{kg} \mathrm{kg}^{-1}$ \\
\hline$q_{1}$ & Initial liquid water mixing ratio in a cloudy volume & $\mathrm{kg} \mathrm{kg}^{-1}$ \\
\hline$q_{\mathrm{v}}$ & water vapor mixing ratio & $\mathrm{kg} \mathrm{kg}^{-1}$ \\
\hline$\widetilde{q}$ & normalized liquid water mixing ratio equal to normalized LWC & nd \\
\hline$r$ & droplet radius & $\mathrm{m}$ \\
\hline$r_{0}$ & initial droplet radius & $\mathrm{m}$ \\
\hline$r_{0}$ & mean droplet radius & $\mathrm{m}$ \\
\hline$r_{\mathrm{V}}$ & mean volume radius & $\mathrm{m}$ \\
\hline$R$ & $\frac{S_{2}}{A_{2} q_{1}}$, potential evaporation parameter $(\mathrm{PEP})$ & nd \\
\hline$R_{\mathrm{a}}$ & specific gas constant of moist air & $\mathrm{J} \mathrm{kg}^{-1} \mathrm{~K}^{-1}$ \\
\hline$R_{\mathrm{V}}$ & specific gas constant of water vapor & $\mathrm{J} \mathrm{kg}^{-1} \mathrm{~K}^{-1}$ \\
\hline$S$ & $e / e_{w}-1$, supersaturation over water & nd \\
\hline$\widetilde{S}$ & normalized supersaturation & nd \\
\hline$S_{2}$ & Initial supersaturation in a dry volume & nd \\
\hline$\widetilde{S}_{\max }$ & maximal normalized supersaturation & nd \\
\hline$T$ & temperature & $\mathrm{K}$ \\
\hline$T_{\text {mix }}$ & normalized duration of inhomogeneous stage & nd \\
\hline$T_{\mathrm{ev}}$ & normalized duration of evaporation & nd \\
\hline$T_{\text {tot }}$ & normalized duration of mixing & nd \\
\hline$t$ & time & $\mathrm{s}$ \\
\hline$\tilde{t}$ & non-dimensional time & nd \\
\hline$x$ & distance & $\mathrm{m}$ \\
\hline$\tilde{x}$ & non-dimensional distance & nd \\
\hline$\lambda_{1}, \quad \lambda_{2}$ & criteria of delimitation between the types of mixing & nd \\
\hline & turbulent dissipation rate & $\mathrm{m}^{2} \mathrm{~s}^{-3}$ \\
\hline$\Gamma(x, t)$ & conservative function & nd \\
\hline$\widetilde{\Gamma}$ & normalized conservative function & nd \\
\hline$\rho_{\mathrm{a}}$ & air density & $\mathrm{kg} \mathrm{m}^{-3}$ \\
\hline$\rho_{\mathrm{W}}$ & density of liquid water & $\mathrm{kg} \mathrm{m}^{-3}$ \\
\hline$\sigma$ & square of droplet radius & $\mathrm{m}^{2}$ \\
\hline$\widetilde{\sigma}$ & normalized square of droplet radius & nd \\
\hline$\tau_{\mathrm{pr}}$ & phase relaxation time & $\mathrm{s}$ \\
\hline$\tilde{\tau}_{\mathrm{pr}}$ & normalized phase relaxation time & nd \\
\hline$\tau_{\operatorname{mix}}$ & characteristic time of mixing & $\mathrm{s}$ \\
\hline$\tau_{0}$ & Initial timescale & $\mathrm{s}$ \\
\hline
\end{tabular}

"nd" denotes non-dimensional. 
Acknowledgements. This research was supported by the Israel Science Foundation (grant 1393/14), the Office of Science (BER), the US Department of Energy Award DE-SC0006788 and the Binational US-Israel Science Foundation (grant 2010446). A. Korolev's participation was supported by Environment Canada.

Edited by: T. Garrett

Reviewed by: two anonymous referees

\section{References}

Andrejczuk, M., Grabowski, W., Malinovski, S. P., and Smolarkiewicz, P. K.: Numerical Simulation of Cloud-Clear Air Interfacial Mixing: Homogeneous versus Inhomogeneous Mixing, J. Atmos. Sci., 66, 2993-2500, 2009.

Baker, M. and Latham, J.: The evolution of droplet spectra and the rate of production of embryonic raindrops in small cumulus clouds, J. Atmos. Sci., 36, 1612-1615, 1979.

Baker, M., Corbin, R. G., and Latham, J.: The influence of entrainment on the evolution of cloud drop spectra: I. A model of inhomogeneous mixing, Q. J. Roy. Meteor. Soc., 106, 581-598, 1980.

Baker, M. B. and Latham, J.: A diffusive model of the turbulent mixing of dry and cloudy air, Q. J. Roy. Meteor. Soc., 108, 871898, 1982.

Burnet, F. and Brenguier, J.-L.: Observational study of the entrainment-mixing process in warm convective cloud, J. Atmos. Sci., 64, 1995-2011, 2007.

Blyth, A. M., Choularton, T. W., Fullarton, G., Latham, J., Mill, C. S., Smith, M. H., and Stromberg, I. M.: The Influence of entrainment on the evolution of cloud droplet spectra. 2. Field experiments 5 at Great Dun Fell, Q. J. Roy. Meteor. Soc., 106, 821-840, 1980.

Boffetta, G. and Sokolov, I. M.: Relative dispersion in fully developed turbulence: The Richardson's law and intermittency correction, Phys. Rev. Lett., 88, 094501, doi:10.1103/PhysRevLett.88.094501, 2002.

Devenish, B. J., Bartello, P., Brenguier, J.-L., Collins, L. R., Grabowski, W. W., Ijzermans, R. H. A., Malinovski, S. P., Reeks, M. W., Vassilicos, J. C., Wang, L.-P., and Warhaft, Z.: Droplet growth in warm turbulent clouds, Q. J. Roy. Meteor. Soc., 138, 1401-1429, 2012.

Gerber, H., Frick, G., Jensen, J. B., and Hudson, J. G.: Entrainment, mixing, and microphysics in trade-wind cumulus, J. Meteorol. Soc. Jpn., 86A, 87-106, 2008.

Hill, A. A., Feingold, G., and Jiang, H.: The Influence of Entrainment and Mixing Assumption on Aerosol-Cloud Interactions in Marine Stratocumulus, J. Atmos. Sci., 66, 1450-1464, 2009.

Jeffery, C. A. and Reisner, J. M.: A study of cloud mixing and evolution using PDF methods. Part I: Cloud front propagation and evaporation, J. Atmos. Sci., 63, 2848-2864, 2006.

Khain, A. P., Ovchinnikov, M., Pinsky, M., Pokrovsky, A., and Krugliak, H.: Notes on the state-of-the-art numerical modeling of cloud microphysics, Atmos. Res., 55, 159-224, 2000.

Korolev, A. and Mazin, I.: Supersaturation of water vapor in clouds, J. Atmos. Sci., 60, 2957-2974, 2003.

Korolev, A., Khain, A., Pinsky, M., and French, J.: Theoretical study of mixing in liquid clouds - Part 1: Classical concepts,
Atmos. Chem. Phys., 16, 9235-9254, doi:10.5194/acp-16-92352016, 2016.

Korolev, A. V.: The influence of suresaturation fluctuations on droplet size spectra formation, J. Atmos. Sci., 52, 3620-3634, 1995.

Kovetz, A. and Olund, B.: The effect of coalescence and condensation on rain formation in a cloud of finite vertical extent, $\mathrm{J}$. Atmos. Sci., 26, 1060-1065, 1969.

Krueger, S., Su, C.-W., and McMurtry, P.: Modeling entrainment and finescale mixing in cumulus clouds, J. Atmos. Sci., 54, 2697-2712, 1997.

Kumar, B., Janetzko, F., Schumacher, J., and Shaw, R. A.: Extremely responses of a coupled scalar-particle system during turbulent mixing, New J. Phys., 14, 115020, doi:10.1088/13672630/14/11/115020, 2012.

Latham, J. and Reed, R. L.: Laboratory studies of effects of mixing on evolution of cloud droplet spectra, Q. J. Roy. Meteor. Soc., 103, 297-306, 1977.

Lehmann, K., Siebert, H., and Shaw, R. A.: Homogeneous and inhomogeneous mixing in cumulus clouds: Dependence on local turbulence structure, J. Atmos. Sci., 66, 3641-3659, 2009.

Lu, C., Liu, Y., Niu, S., and Endo, S.: Scale dependence of entrainment-mixing mechanisms in cumulus clouds, J. Geophys. Res.-Atmos., 119, 13877-13890, doi:10.1002/2014JD022265, 2014.

Magaritz-Ronen, L., Khain, A., and Pinsky, M.: About the Horizontal Variability of Effective Radius in Stratocumulus Clouds, J. Geophys. Res., in review, 2016.

Martin, G. M., Johnson, D. W., and Spice, A.: The measurements and parameterization of effective radius of droplets in warm stratocumulus clouds, J. Atmos. Sci., 51, 1823-1842, 1994.

Monin, A. S. and Yaglom, A. M.: "Statistical Fluid Mechanics: Mechanics of Turbulence", Vol. 2, MIT Press, 1975.

Morrison, H. and Grabowski, W. W.: Modeling supersaturation and subgrid-scale mixing with two-moment bulk warm microphysics, J. Atmos. Sci., 65, 792-812, 2008.

Pinsky, M. and Khain, A. P.: Effects of in-cloud nucleation and turbulence on droplet spectrum formation in cumulus clouds, Q. J. Roy. Meteor. Soc., 128, 1-33, 2002.

Pinsky, M., Mazin, I. P., Korolev, A., and Khain, A.: Supersaturation and diffusional droplet growth in liquid clouds, J. Atmos. Sci., 70, 2778-2793, 2013.

Pinsky, M., Mazin, I. P., Korolev, A., and Khain, A.: Supersaturation and diffusional droplet growth in liquid clouds: Polydisperse spectra, J. Geophys. Res.-Atmos., 119, 12872-12887, 2014.

Pinsky, M., Khain, A., Korolev, A., and Magaritz-Ronen, L.: Theoretical investigation of mixing in warm clouds - Part 2: Homogeneous mixing, Atmos. Chem. Phys., 16, 9255-9272, doi:10.5194/acp-16-9255-2016, 2016.

Polyanin, A. D. and Zaitsev, V. F.: Handbook of nonlinear partial differential equations, Chapman \& Hall/CRC, 809 pp., 2004

Prabha, V. T., Patade, S., Pandithurai, G., Khain, A., Axisam, D., Pradeep Kumar, P., Maheshkumar, R. S., Kulkarni, J. R., and Goswami, B. N.: Spectral width of premonsoon and monsoon clouds over Indo-Gangetic valley during CAIPEEX, J. Geop. Res., 117, D20205, doi:10.1029/2011JD016837, 2012.

Pruppacher, H. R. and Klett, J. D.: Microphysics of Clouds and Precipitation, 2nd Edn., Oxford Press, 914 pp., 1997. 
Rogers, R. R. and Yau, M. K.: A Short Course in Cloud Physics, Pergamon press, 293 pp., 1989.

Schlüter, M. H.: The effects of entrainment and mixing process on the droplet size distribution in cumuli. A thesis submitted to the faculty of The University of Utah in partial fulfillment of the requirements for the degree of Master of Science, Department of Meteorology, The University of Utah, 92 pp., 2006.
Su, C.-W., Krueger, S. K., McMurtry, P. A., and Austin, P. H.: Linear eddy modeling of droplet spectral evolution during entrainment and mixing in cumulus clouds, Atmos. Res., 47-48, 41-58, 1998.

Warner, J.: The microstructure of cumulus cloud: Part 4: The effect on the droplet spectrum of mixing between cloud and environment, J. Atmos. Sci. 30, 256-261, 1973. 